\title{
Diffusion of water and selected atoms in DMPC lipid bilayer membranes
}

\author{
Hansen, Flemming Yssing; Peters, Günther H.J.; Taub, H.; Miskowiec, A.
}

Published in:

Journal of Chemical Physics

Link to article, DOI:

$10.1063 / 1.4767568$

Publication date:

2012

Document Version

Publisher's PDF, also known as Version of record

Link back to DTU Orbit

Citation (APA):

Hansen, F. Y., Peters, G. H. J., Taub, H., \& Miskowiec, A. (2012). Diffusion of water and selected atoms in DMPC lipid bilayer membranes. Journal of Chemical Physics, 137(20), Paper 204910.

https://doi.org/10.1063/1.4767568

\section{General rights}

Copyright and moral rights for the publications made accessible in the public portal are retained by the authors and/or other copyright owners and it is a condition of accessing publications that users recognise and abide by the legal requirements associated with these rights.

- Users may download and print one copy of any publication from the public portal for the purpose of private study or research.

- You may not further distribute the material or use it for any profit-making activity or commercial gain

- You may freely distribute the URL identifying the publication in the public portal

If you believe that this document breaches copyright please contact us providing details, and we will remove access to the work immediately and investigate your claim 


\section{AIP $\begin{gathered}\text { mesoumalor } \\ \text { Chemical Physics }\end{gathered}$}

Diffusion of water and selected atoms in DMPC lipid bilayer membranes

F. Y. Hansen, G. H. Peters, H. Taub, and A. Miskowiec

Citation: J. Chem. Phys. 137, 204910 (2012); doi: 10.1063/1.4767568

View online: http://dx.doi.org/10.1063/1.4767568

View Table of Contents: http://jcp.aip.org/resource/1/JCPSA6/v137/i20

Published by the American Institute of Physics.

Additional information on J. Chem. Phys.

Journal Homepage: http://jcp.aip.org/

Journal Information: http://jcp.aip.org/about/about_the_journal

Top downloads: http://jcp.aip.org/features/most_downloaded

Information for Authors: http://jcp.aip.org/authors

\section{ADVERTISEMENT}
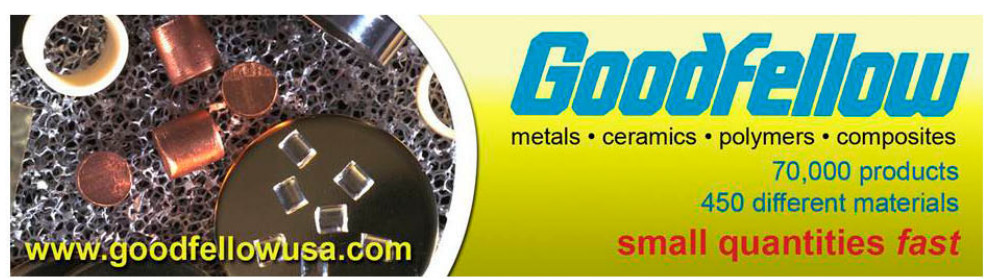


\title{
Diffusion of water and selected atoms in DMPC lipid bilayer membranes
}

\author{
F. Y. Hansen, ${ }^{1, a)}$ G. H. Peters, ${ }^{1}$ H. Taub, ${ }^{2}$ and A. Miskowiec ${ }^{2}$ \\ ${ }^{1}$ Department of Chemistry, Technical University of Denmark, IK 207 DTU, DK-2800 Lyngby, Denmark \\ ${ }^{2}$ Department of Physics and Astronomy and the University of Missouri Research Reactor, \\ University of Missouri, Columbia, Missouri 65211, USA
}

(Received 21 May 2012; accepted 1 November 2012; published online 29 November 2012)

\begin{abstract}
Molecular dynamics simulations have been used to determine the diffusion of water molecules as a function of their position in a fully hydrated freestanding 1,2-dimyristoyl-sn-glycero-3phosphorylcholine (DMPC) bilayer membrane at $303 \mathrm{~K}$ and $1 \mathrm{~atm}$. The diffusion rate of water in a $\sim 10 \AA$ thick layer just outside the membrane surface is reduced on average by a factor of $\sim 2$ relative to bulk. For water molecules penetrating deeper into the membrane, there is an increasing reduction in the average diffusion rate with up to one order of magnitude decrease for those deepest in the membrane. A comparison with the diffusion rate of selected atoms in the lipid molecules shows that $\sim 6$ water molecules per lipid molecule move on the same time scale as the lipids and may therefore be considered to be tightly bound to them. The quasielastic neutron scattering functions for water and selected atoms in the lipid molecule have been simulated and compared to observed quasielastic neutron scattering spectra from single-supported bilayer DMPC membranes. (C) 2012 American Institute of Physics. [http://dx.doi.org/10.1063/1.4767568]
\end{abstract}

\section{INTRODUCTION}

The hydration of biological cell membranes is necessary for their proper functioning. It is therefore important to understand the structural and dynamic properties of water near and inside the membranes. Different experimental techniques have been employed to study the structure and dynamics of water at the surface of a membrane such as NMR, ${ }^{1-3}$ time-resolved fluorescence spectroscopy, ${ }^{4,5}$ neutron scattering, ${ }^{6-11}$ ultrafast spectroscopy, ${ }^{12}$ and IR absorption spectroscopy. ${ }^{13}$ Computer simulation techniques such as Monte Carlo and molecular dynamics (MD) have also been applied to study structural and dynamic properties of water near membranes. ${ }^{14-17}$

In their study of hydration water by ultrafast terahertz time-domain spectroscopy, Hishada and Tanaka ${ }^{12}$ note that the effect of the lipid molecules on the water dynamics depends on the time scale of the water motion. They studied the ultrafast rotational relaxation dynamics of water molecules $\left(\sim 10^{-13} \mathrm{~s}\right)$ in a water-DMPC (1,2-dimyristoyl-sn-glycero3 - phosphorylcholine) lipid system and found a long-range hydration effect up to 4-5 layers of water $(\sim 10 \AA)$ above the surface of the phospholipid bilayer. This result contrasted with earlier findings by NMR and neutron scattering measurements ${ }^{1,3,6}$ on time scales of $\sim 10^{-6}-10^{-11}$ s that showed only a single layer of relatively slow-moving water molecules on the membrane surface to be affected by the lipids. The faster water molecules further away from its surface were missed by these other techniques. This observed long-range hydration effect is consistent with previous predictions by MD simulations. ${ }^{17-19}$ The time step in such simulations is typically about $10^{-15} \mathrm{~s}$, so phenomena on a time

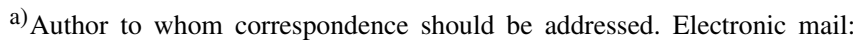
flemming@kemi.dtu.dk.
}

scale of $10^{-13}-10^{-12} \mathrm{~s}$, as in terahertz spectroscopy, may be studied.

MD simulations ${ }^{14,15}$ have also been used to study the interfacial structure, the ordering of water, and its relation to the hydration force in membranes of the lipid dipalmitoylphosphatidyl-choline (DPPC). The mobility of water and lipid molecules has been determined both on a short time scale of $\sim 10-20 \mathrm{ps}$ and on a longer time scale of $\sim 200 \mathrm{ps}$. The simulations show that the electric field caused by charges on the atoms in the head group extends $\sim 10-15 \AA$ outside the membrane consistent with the claim that hydration effects extend out to a distance of $\sim 10 \AA$ from the surface of the membrane. ${ }^{12}$

Here we report MD simulations of a freestanding DMPC lipid bilayer system for which we have studied both the interfacial structure and the dynamics of water and lipid molecules. Our particular focus is on slow translational diffusive motion on the time scale $10^{-9}-10^{-11} \mathrm{~s}$. A strong motivation for these simulations has been our series of highenergy-resolution quasielastic incoherent neutron scattering experiments ${ }^{20}$ which have been used to elucidate the diffusion of water molecules in proximity to a single-supported DMPC membrane. By varying sample temperature and level of hydration and using partial deuteration of the hydrogen atoms, we were able to identify three different time scales for the diffusive motion of water: a fast one as in bulk water, a slower one attributed to water confined by the membrane, and a very slow one, comparable to the nanosecond time scale for the motion of $\mathrm{H}$ atoms in the lipids. This slowest motion we identify with water molecules strongly bound to the lipid molecules.

In our MD simulations, we have calculated the quasielastic incoherent neutron scattering functions for comparison with experiments. In addition, we have used the MD simulations to determine the density profile of water in the 
membrane and to determine the diffusion constant of water as a function of its depth in the membrane. This information is not directly available in scattering experiments which include contributions from all water molecules irrespective of their position. The experimental results are some weighted average of the different diffusion rates of the water molecules in the membrane. But, if the time scale for the motion of some water molecules is sufficiently different from the motion of others, the different time scales may be resolved in the experiments. The binding of water to the lipid molecules has also been studied, and the relative amount of "loosely" and "strongly" bound water molecules have been estimated.

There is one important limitation to our comparison of the neutron experiments and simulations, because, for practical reasons, they were done on somewhat different systems. To conduct the experiments under controlled conditions, it was necessary to deposit the single bilayer membrane on a solid substrate, a single-crystal silicon wafer. To obtain enough intensity of scattered neutrons, we used a stack of 100 silicon wafers, coated with a single bilayer membrane on each side. Other neutron scattering experiments ${ }^{11}$ have used larger samples consisting of stacks of a thousand or more membranes supported on a solid substrate in order to build up sufficient scattered neutron intensity. These samples reduce the contribution from the perturbed leaflet adjacent to the solid substrate, but the sheer number of membranes, the interaction between them within a stack, and the presence of unknown amounts of water between membranes complicates the simulation of these samples and the comparison with experiments.

We have chosen to begin with MD simulations of a single freestanding bilayer membrane because it is simpler than a single-supported membrane and because it will be a good reference for identifying the effects of the substrate in a planned simulation of a single-supported membrane. Some recent neutron reflectivity measurements ${ }^{21}$ indicate that the water density profile in the upper leaflet of the adsorbed bilayer membrane is similar to that found in simulations of a freestanding bilayer membrane, while it is perturbed in the leaflet adjacent to the substrate.

Our work extends previous MD simulations on membrane systems in essentially four ways: (1) By combining a calculation of the quasielastic spectra for water with those for selected atoms in the lipids, it has been possible to determine the number of tightly bound water molecules to the lipids. The energy resolution and dynamical range in the calculations have been chosen to be the same as in quasielastic neutron scattering experiments on single-supported bilayer membranes which makes a detailed comparison between experiments and simulations possible. (2) By dividing the membrane region into slabs parallel to the surface, it has been possible to determine the mobility of water molecules as a function of their depth in the membrane. The results show that there is a significant reduction in the mobility as we move deeper into the membrane. (3) A kinetic analysis of the water molecules bound in the first solvation shell around polar groups in the lipids shows that there are three bound states; the binding strength changes by an order of magnitude from one state to the other. The kinetic analysis yields a more qualitative estimate of the number of strongly bound water molecules in fair agreement with the number derived from the simulated quasielastic neutron spectra. (4) The importance of the length of the time-window for the diffusion constant determined in a mean-square displacement calculation is discussed and analyzed when bounded motions, like rotations and librations, are involved in addition to unbounded motions.

This paper is organized into nine sections. Section II has some details about the MD simulations and treatment of the data before they are analyzed. Section III contains calculations of the atomic density profiles in the direction perpendicular to the membrane surface. Section IV follows with an analysis of the diffusion of water molecules as a function of their position in the membrane. For comparison, we have also included results from our simulations on pure bulk water. Section $\mathrm{V}$ contains an analysis of the diffusion of selected atoms in the lipid molecules. In Sec. VI, we present a kinetic analysis of the binding of water to selected atoms in the lipid molecules. Section VII has a calculation of both the intermediate quasielastic incoherent neutron scattering function and the quasielastic incoherent scattering function, which is proportional to the scattered intensity from the sample. Section VIII has comparisons with experiments; and, finally, Sec. IX has our conclusions.

\section{MD SIMULATIONS AND DATA TREATMENT}

The simulated system is a single, free, bilayer membrane with 64 DMPC molecules in each leaflet, a total of 128 DMPC molecules, and 4952 water molecules at a temperature of $303 \mathrm{~K}$ and a pressure of $1 \mathrm{~atm}$. The system has a total of 38.7 water molecules per lipid, which is larger than the $\sim 29$ water molecules per lipid required for the membrane to be fully hydrated. $^{29}$

The system is modeled using a slightly modified version of the CHARMM27 force field ${ }^{22}$ where some of the charges in the head group region have been altered because the calculated area per lipid is too small using the original force field. We implemented the modification developed by Sonne et al. ${ }^{23}$ for the DPPC bilayer membrane system, assuming that the results carried over to the DMPC bilayer system. After having completed this work, we became aware of a new force field for DMPC, ${ }^{24}$ which gives an excellent value of the area/lipid as will be discussed further below. For water, we have used the TIP3P force field ${ }^{25}$ and done the simulations with water molecules in which both bonds and bond angle are flexible. The simulations were done with the NAMD program package, ${ }^{26}$ using a time step of $1 \mathrm{fs}$ and recording data every $0.5 \mathrm{ps}$ for later analysis. The sampling was done in the NPT ensemble; the target temperature of the Langevin thermostat was $303 \mathrm{~K}$ with a damping coefficient of $5 \mathrm{ps}^{-1}$. The pressure was controlled by a Nosé-Hoover Langevin barostat ${ }^{27}$ with a piston oscillation time of $200 \mathrm{fs}$ and a damping time of $500 \mathrm{ps}$. Anisotropic pressure regulation was applied in the simulations of the membrane system, while isotropic pressure regulation was used in the simulation of bulk water. The long-range electrostatic forces were calculated using the particle mesh Ewald method ${ }^{28}$ with a $\sim 1 \AA$ grid spacing, and the interpolation was done with a fourth-order spline fit. Electrostatic forces were updated every fourth femto second. 


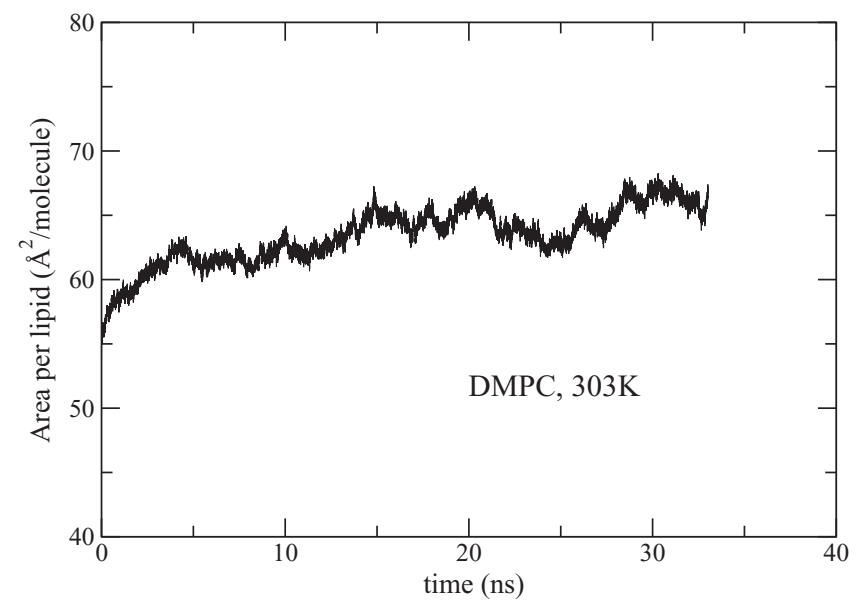

FIG. 1. The area/lipid in the simulation of the DMPC lipid bilayer membrane as a function of time at a temperature of $303 \mathrm{~K}$ and 1 atm of pressure. The area is estimated to be $64.9 \pm 1.4 \AA^{2}$ when the system is equilibrated. The area estimated from $x$-ray experiments ranges from $59.6 \AA^{2}$ (Ref. 29) to $60.6 \AA^{2} .30$

Van der Waals interactions were cut off at $12 \AA$ in combination with a switching function starting at $10 \AA$. Periodic boundary conditions were used in all three Cartesian directions; the water layer at each surface of the membrane is about $10 \AA$ thick which is sufficient to eliminate any artificial interaction in the $z$-direction between headgroups in the upper and lower leaflets. The size of the simulation box fluctuates with time because the sampling is done in the NPT ensemble, and its dimensions are approximately, $x_{\text {box }} \sim 70 \AA, y_{\text {box }}$ $\sim 60 \AA$, and $z_{\text {box }} \sim 70 \AA$. The temperature was chosen to be above the gel-to-fluid transition temperature $\sim 297 \mathrm{~K}$ because we wanted to study the system in the fluid state, which is the biologically relevant phase. A calculation of the NMR order parameter (not shown) confirmed that the system is in the fluid phase.

The setup was an equilibrated system (60 ns) from a previous simulation with the original CHARMM27 force field. For this reason, we see an initial increase in the area/lipid in Fig. 1 where we have plotted the area/lipid as function of time. The system was simulated for 34 ns with the modified force field; and, after about $10 \mathrm{~ns}$, the area/lipid levels out and oscillates about a nearly constant value estimated to be $64.9 \pm 1.4 \AA^{2}$. This value is significantly larger than $\sim 57 \AA^{2}$ obtained with the original force field and also somewhat larger than determined by $\mathrm{X}$-ray experiments, $\sim 59.6 \AA^{229}$ and $\sim 60.6 \AA^{2} .^{30}$ This discrepancy indicates that the transfer of the optimized charges from the DPPC membrane system is only partially justified. Because the area/lipid is only $7 \%-8 \%$ larger $\left(\sim 4 \AA^{2}\right)$ than inferred by x-ray measurements, we do not expect this structure to allow penetration of significantly more water than in the membrane with the smaller area/lipid. In fact, our water density profile agrees well with other simulations. ${ }^{14,15}$ This feature combined with our membrane being in the fluid phase indicates that a simulation with the new force field ${ }^{24}$ will probably give results that are qualitatively similar to our own.

The computation has been broken up into time blocks of $3.5 \mathrm{~ns}$; and we have used the data in time block $24.5-28 \mathrm{~ns}$ in our analysis. A time block of 3.5 ns corresponds to an energy resolution of $1.2 \mu \mathrm{eV}$ which is better than the $3.5 \mu \mathrm{eV}$ of the BASIS spectrometer. Therefore, it is of sufficient length for obtaining results with the same energy resolution as in the experiments. $^{20}$ The box dimensions and atomic coordinates are recorded every $0.5 \mathrm{ps}$, which gives 7000 frames in each time block. The 0.5 ps sampling-time is the best time resolution in the simulations and corresponds to a maximum frequency of $1 /(2 \times 0.5)=1 \mathrm{THz}$ or a dynamical range of $\sim 4.13 \mathrm{meV}$. This range is larger than the $125 \mu \mathrm{eV}$ dynamical range of the BASIS spectrometer used in the quasielastic neutron scattering experiments. ${ }^{20}$ We may do an analysis with a poorer time resolution (a smaller dynamical range) by skipping frames in the analysis of the time dependence of a quantity. In summary, both the time-window and time-resolution in the simulations are sufficient for a comparison of simulated and experimental scattering functions.

Because we are going to analyze the diffusion of molecules and atoms in different regions of the membrane in the $z$-direction, perpendicular to the membrane plane, it is important that there be no overall motion or drift of the system in this direction. If the center-of-mass position of the entire system is placed at the center of the simulation box located at the origin of the coordinate system and the center-of-mass velocity is zero in the setup of the simulation, then the centerof-mass position should remain at the origin at all times. In practice, however, we found a drift in the center-of-mass position which is probably an effect of both numerical round-off errors and the application of a thermostat. This phenomenon is well-known in constraint dynamics simulations where the satisfaction of the constraints becomes poorer and poorer as the simulation progresses because of numerical round-off errors. The solution to the problem is to ensure that the constraints are satisfied at each time step. Similar measures could be taken to require that the center-of-mass position at the origin of the coordinate system does not drift; but due to an oversight, the flag for ensuring this condition was not set in our simulations.

Instead, we have corrected for the drift by determining the $z$-coordinate of the center-of mass position in each time frame and subtracted it from the $z$-coordinates of all atoms. In this way, the drift of the membrane system in the $z$-direction was eliminated. All atoms are kept in the simulation box so that we have allowed for the periodic boundary conditions when unfolding trajectories for evaluating the diffusion constants.

\section{ATOMIC DENSITY PROFILES ALONG THE z-DIRECTION}

We have determined the distribution of water molecules and selected lipid-molecule atoms in the $z$-direction, perpendicular to the membrane surface. The five selected atoms are: (1) the nitrogen atom in the choline unit in the head group; (2) the phosphorous atom in the phosphate unit in the head group; (3) the center carbon atom in the glycerol backbone; (4) the carbonyl carbon atom at the link between the aliphatic chain and the glycerol; and (5) the carbon atom in the methyl group at the end of the aliphatic chains. These selected atoms 

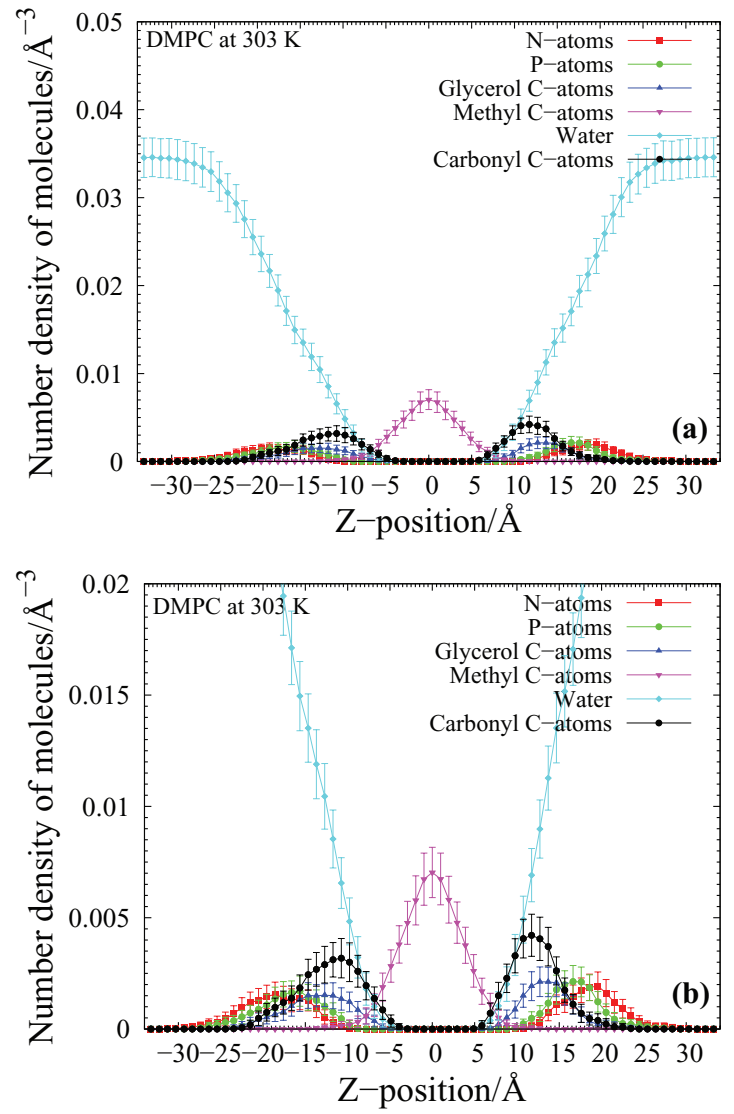

FIG. 2. (a) The number density profile along the $z$-axis, perpendicular to the membrane surface, for a number of selected lipid-molecule atoms and water molecules in a DMPC lipid bilayer membrane at $303 \mathrm{~K}$ and $1 \mathrm{~atm}$ pressure. (b) A blow-up of (a) to make the profiles inside the membrane more distinct. The membrane thickness, measured as the distance between the peak positions of the nitrogen atoms, is $\sim 36 \AA$. It is probably an underestimate because of the methyl groups attached to the nitrogen atoms. The very rough surface of the membrane makes it difficult to estimate its thickness. Experimentally, it may depend on the probe used to evaluate it.

in the lipid molecule encompass the molecule's extent in the $z$-direction.

The space along the $z$-axis of the simulation box has been divided into 69 layers which have been defined to be symmetric about the membrane's midpoint with the center layer located at $z=0$. From the average box size, the average width of the layers is $\Delta z_{\text {layer }}=0.97763 \pm 0.0144 \AA$. The $z$-coordinates of the water molecules and selected atoms were used to assign them to one of the 69 layers; and the number of molecules and atoms in each layer was accumulated for all 7000 time frames. The final number of molecules in each layer was found as an average over all frames. The results were converted to a number density profile by dividing the number of atoms in each layer by its volume.

The number density of water molecules and selected atoms along the $z$-direction are shown in Fig. 2. Because the number density of water molecules near the surface of the membrane is much larger than that of the selected atoms, we have shown in Fig. 2(b) a blow-up of the distribution in Fig. 2(a) to clarify details of the membrane interior. We notice that the distributions appear symmetric about $z=0$, the center of the bilayer membrane, which implies that we have

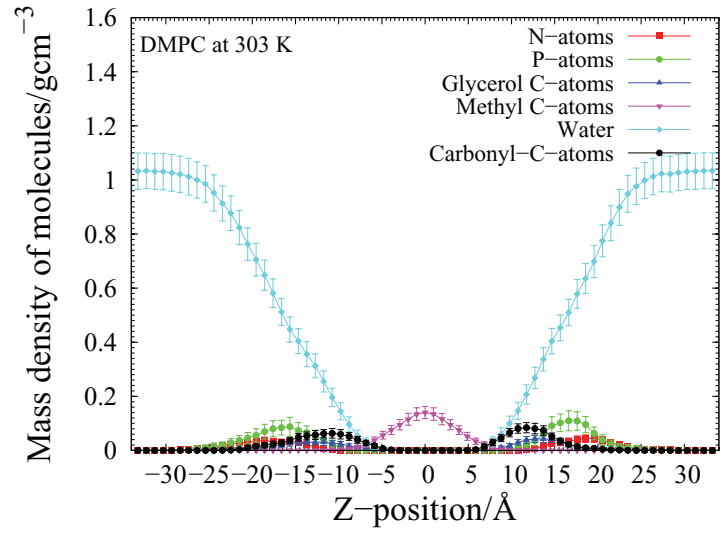

FIG. 3. The mass-density profile along the $z$-axis, perpendicular to the membrane surface, for a number of selected atoms in the lipid molecule and for water molecules in a DMPC lipid bilayer membrane at $303 \mathrm{~K}$ and $1 \mathrm{~atm}$ pressure.

been successful in removing most of the membrane's centerof-mass drift. In principle, there should be perfect symmetry because the two leaflets are identical; the slight difference is probably related to the statistical uncertainty.

The water profile shows an almost constant number density outside the membrane in the $z$-range $\sim \pm(25-35) \AA$. Therefore, we interpret these regions to be bulk-like water, a proposition that is supported by the mass density. It is determined from the number density by multiplication of the molar and atomic masses and is plotted in Fig. 3. For water, it is seen to be $\sim 1.03 \mathrm{~g} \mathrm{~cm}^{-3}$, which is close to the experimental value at $303 \mathrm{~K}$ of $0.9956488 \mathrm{~g} \mathrm{~cm}^{-331}$ and is identical to the density in our simulation of bulk water, $\sim 1.03 \mathrm{~g} \mathrm{~cm}^{-3}$ at $303 \mathrm{~K}$ and $1 \mathrm{~atm}$. We deliberately refer to it as bulk-like water, because the dynamics of the water molecules in these regions outside the membrane is different from bulk as we shall discuss below.

The penetration of the water molecules into the membrane is quite substantial and extends down to the region of the carbonyl groups. From $\sim \pm 10 \AA$ down to $\sim \pm 5 \AA$, the density of the water molecules follows that of the lipid carbonyl groups very closely, which may indicate that the water molecules in that region are tightly bound to the carbonyl groups. This possibility is supported by our analysis of the water molecule dynamics to be discussed below.

A general feature of the number density distribution functions for all the selected atoms is that they are quite broad with a full-width-at-half-maximum (FWHM) $\sim 7-8 \AA$, a result which is consistent with simulation results on the DPPC membrane system with the same head group as DMPC. ${ }^{14-16}$ Experimental determinations using supported membranes have a root-mean-square amplitude of the perpendicular $(z)$ motion of the lipids in the range 2-3 $\AA,,^{10,32}$ which is equivalent to a FWHM of 4-6 $\AA$ and in good agreement with the simulation results. From Fig. 2(b), it is clear that there is even a small overlap between the distribution function for the methyl groups at the end of the hydrophobic aliphatic chains and the hydrophilic carbonyl groups. The overlap depends on the length of the aliphatic chains. If they are relatively short, as in the case of DMPC with 14 carbon atoms, the overlap is 
TABLE I. Definition of the layers in a DMPC bilayer membrane at a temperature of $303 \mathrm{~K}$ and a pressure of $1 \mathrm{~atm}$ as used in the study of the water dynamics as a function of the position in the membrane. The designation of the layers is motivated by the density profile in Fig. 2.

\begin{tabular}{lccr}
\hline \hline Layer number & $\begin{array}{c}\text { Mean position } \\
z_{\mathrm{m}} / \AA\end{array}$ & $\begin{array}{c}\text { Lower limit } \\
z_{\min } / \AA\end{array}$ & $\begin{array}{c}\text { Upper limit } \\
z_{\max } / \AA\end{array}$ \\
\hline 1 (bulk-like water) & -30.25 & -35.50 & -25.0 \\
2 (outer membrane) & -21.00 & -25.00 & -17.00 \\
3 (inner membrane) & -8.50 & -17.00 & 0.00 \\
4 (inner membrane) & 8.50 & 0.00 & 17.00 \\
5 (outer membrane) & 21.00 & 17.00 & 25.00 \\
6 (bulk-like water) & 30.25 & 25.00 & 35.50 \\
\hline \hline
\end{tabular}

larger than in our simulations of the DOPC bilayer membrane (results not shown) with the same headgroup and 18 carbon atoms in the aliphatic chains.

The position of the maximum in the distribution functions for the nitrogen and phosphorous atoms are only a few Ångstrom apart, which implies that the line between them or the dipole that they form is almost parallel to the membrane surface. The distance between the maxima of the nitrogen atom distribution functions in the two leaflets is $\sim 36 \AA$. It may be taken as a lower limit of the membrane thickness, because the methyl groups attached to the nitrogen atoms add to the thickness by 1-2 $\AA$. This thickness is in fair agreement with an x-ray estimate of $\sim 37 \AA .{ }^{33}$

\section{DIFFUSION OF WATER MOLECULES}

The diffusion of water molecules in the DMPC lipid bilayer membrane has been studied by calculating several dynamical properties: (1) the transition probabilities for water molecules to move along $z$ from one layer (the layers are defined below) to another in the membrane; (2) the average residence times for water molecules in the different layers; (3) the mean-square displacements and diffusion constants for the water molecules within each of the layers; (4) the mean-square displacement and diffusion constant for all water molecules irrespective of their positions in the membrane for comparison with the neutron scattering experiments.

For our dynamical studies, we used a coarser layer division than for the atomic density profile along the $z$-direction and found it natural to divide the $z$-region for each leaflet into three layers: (1) a layer outside the membrane with bulk-like water; (2) an outer layer around the nitrogen and phosphorous atoms in the head group; and (3) an inner layer with the glycerol and carbonyl groups and aliphatic chains. The delimiters of these layers are given in Table I and are based on the number-density distributions in Fig. 2.

For a consistent comparison between the translational motion of the water molecules in the membrane and in bulk, we have also done simulations of bulk water using the same force model $^{25}$ as in the membrane simulations.

\section{A. Transition probabilities and residence times}

The $z$ position of each water molecule was monitored as a function of time in a given time-window. There are two possibilities: (1) the molecule stays in the layer where it started for the entire length of time set by the time-window; (2) the molecule leaves the layer it started in after some time and moves to an adjacent layer. The length of time each molecule stayed in the layer where it started is monitored together with the layer to which it eventually moved in the given timewindow. These results, averaged over all molecules within the layers, are used to determine transition probabilities and residence times. The results of the calculations with a 1 ns timewindow and a 5 ps time resolution are compiled in Table II.

The $(i, j)$ element in the $(6 \times 6)$ matrix shows the probability in \% for a transition of a water molecule from layer $i$ to layer $j$ within the given time-window. The matrix has "two blocks" along the diagonal, one for each leaflet. For example, we see that the $(1,1)$ element is $1 \%$, which means that the transition probability from layer one to layer one, or equivalently, the probability for staying in layer one for a time equal to the length of the time-window, is $1 \%$. The probability for a transition from layer one to layer 2 is given by element $(1,2)$ which is equal to $46.1 \%$. No molecules from layer one make it directly to layer $3,4,5$ because the elements $(1,3),(1,4)$, and $(1,5)$ are equal to zero, while the transition probability from layer 1 to layer 6 , both with bulk-like water, is $52.9 \%$. This transition is a result of the periodic boundary conditions and is, of course, not through the membrane.

The average residence time for molecules in layer one is 31 ps; i.e., a molecule stays on average in layer one for $31 \mathrm{ps}$ before it moves to either layer 2 or layer 6 . The $3 \times 3$ blocks around the diagonal are similar as are the $3 \times 3$ off-diagonal blocks, displaying the symmetry of the leaflets. This symmetry is also reflected in the average residence times for equivalent layers.

It should be realized that the calculated average residence times may depend on the length of the time-window used in the calculations. If a residence time is longer than the

TABLE II. Transition probability (in \%) between layers (defined in Table I) and average residence time in layers for water molecules in a DMPC lipid bilayer membrane at $303 \mathrm{~K}$ and $1 \mathrm{~atm}$, using a $1 \mathrm{~ns}$ time-window and a time resolution of $5 \mathrm{ps}$.

\begin{tabular}{lrrrrrrr}
\hline \hline$i \backslash j$ & 1 & 2 & 3 & 4 & 5 & 6 & Av. residence time/ps \\
\hline 1 (bulk-like water) & 1.00 & 46.09 & 0.00 & 0.00 & 0.00 & 52.91 & 31.00 \\
2 (outer membrane) & 66.89 & 1.58 & 31.53 & 0.00 & 0.00 & 0.0008 & 53.53 \\
3 (inner membrane) & 0.00 & 84.49 & 15.43 & 0.08 & 0.00 & 0.00 & 255.20 \\
4 (inner membrane) & 0.00 & 0.00 & 0.00 & 12.97 & 87.03 & 0.00 & 58.23 \\
5 (outer membrane) & 0.00 & 0.00 & 0.00 & 30.25 & 1.53 & 0.91 & 54.26 \\
6 (bulk-like water) & 52.28 & 0.003 & 0.00 & 0.00 & 46.82 & & 30.16 \\
\hline
\end{tabular}


time-window, we will obviously determine too small a residence time. A simple analysis (not included here) shows that, if a residence time is 6-7 times smaller than the time-window, then the calculated residence time will be accurate at the $1 \%$ level. For longer residence times, the calculated value will be smaller than the true value. We see that the residence times in Table II satisfy the condition for determining an accurate residence time except for the largest residence times which may be slightly smaller than the true values. The time resolution may also have an effect on the average residence times but that will be negligible compared to the effect of the length of the time-window.

Both residence times and transition probabilities clearly show a significant decrease in the interlayer exchange of the water molecules as we enter deeper into the membrane. This decrease is probably related to a stronger binding of the water molecules to the lipids deeper into the membrane.

\section{B. Mean-square displacements of water molecules}

The calculation of the mean-square displacements for the water molecules in the different layers in the membrane is done in two steps. When a time-origin has been chosen, each water molecule is assigned to a layer according to its $z$-coordinate and labeled with a "layer-tag." We then follow each molecule in time for the chosen time-window. Only those molecules which stay in the same layer for the entire timewindow are used in the determination of the mean-square displacement of the water molecules in that particular layer. This procedure sets an upper limit for the time-window that may be used because, at sufficiently long times, no molecules remain in the layer in which they started. Therefore, it is essential that the "diffusion limit," the linear relationship between mean-square displacement and time, is reached within a time that is shorter than the upper limit for the time-window.

In our analysis, we have used a time-window of $100 \mathrm{ps,}$ which we find to be sufficient to establish a linear relationship between the mean-square displacement and time. The meansquare displacements have been averaged over all molecules remaining in the given layer for the entire time-window and over 700 choices of time origins. Before the determination of the displacement, we checked whether a given molecule has been moved by a box dimension due to the periodic boundary conditions. In this case, the displacement is corrected for that unphysical motion.

The mean-square displacements of the water molecules in the lower leaflet are shown as function of time in Fig. 4. Similar results are obtained for the upper leaflet (not shown), which should be equivalent. We note that the mean-square displacement curves are linear in time after $\sim 20$ ps. From the slope $\alpha$ of the linear part of the curves in the time range $20<t<100 \mathrm{ps}$, a diffusion constant $D=\alpha / 6$ is calculated. ${ }^{34}$ The results are summarized in Table III. They show that there is an order of magnitude difference between the diffusion constant for water in the bulk-like water layer outside the membrane and the inner membrane layer with an intermediate diffusion constant for water molecules in the outer layer of the membrane. We see that the reduction in the interlayer transi-

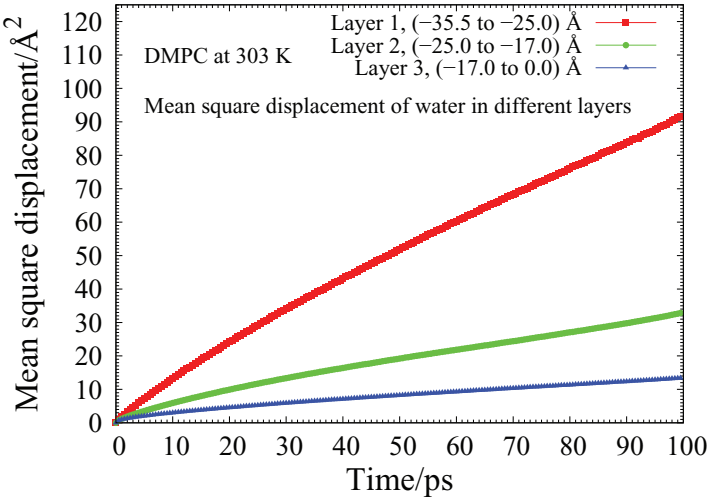

FIG. 4. The mean-square displacements of water molecules in each of the three layers defined in Table I in the lower leaflet of the DMPC membrane at $303 \mathrm{~K}$ and $1 \mathrm{~atm}$. These calculations use a $100 \mathrm{ps}$ time-window and a $0.5 \mathrm{ps}$ time-resolution.

tion probability for water molecules as we go deeper into the membrane correlates with a reduced rate of diffusion. A combination of different effects is probably responsible for this reduction in the diffusion rate; e. g., the density of "foreign" atoms from the lipids, electrostatic effects, and the binding of water molecules to polar groups in the lipid molecules.

The mean-square displacement of all water molecules, irrespective of their positions in the membrane, has also been calculated and gives a diffusion constant of $D=1.05$ $\times 10^{-5} \mathrm{~cm}^{2} \mathrm{~s}^{-1}$. This value compares well with the diffusion constant $D=9.65 \times 10^{-6} \mathrm{~cm}^{2} \mathrm{~s}^{-1}$ calculated as a molefraction weighted average of the diffusion constants for water molecules in the different layers. The mole-fractions were estimated from the number-density profile of water in Fig. 2.

To clarify the effect of the membrane on the translational diffusion of water molecules, we have determined the diffusion constant for water molecules in a simulation of bulk water using the same force model as in the membrane simulations. The simulations have been done on a system of 2080 water molecules at $303 \mathrm{~K}$ and $1 \mathrm{~atm}$. The system has been simulated for $10 \mathrm{~ns}$, and we have used a time block of $2.4 \mathrm{~ns}$ in the analysis with a well-equilibrated system. The simulation gives a water density $\rho=1.033 \mathrm{~g} \mathrm{~cm}^{-3}$, which is the same density as the bulk-like layer in the membrane simulations. The mean-square displacements are determined in a 100 ps time-window by averaging over 100 randomly

TABLE III. The slope $\alpha$ of the linear part ( $20<t<100 \mathrm{ps)}$ of the meansquare displacement curves in Fig. 4 and diffusion constants $(D=\alpha / 6)$ for water molecules in the different layers in the DMPC membrane at $303 \mathrm{~K}$ and $1 \mathrm{~atm}$, using a $100 \mathrm{ps}$ time-window and a $0.5 \mathrm{ps}$ time resolution. The definition of the layers is given in Table I. The experimental and simulated diffusion constant for bulk water is $2.33 \times 10^{-5} \mathrm{~cm}^{2} \mathrm{~s}^{-1}$.

\begin{tabular}{lcc}
\hline \hline Layer & $\alpha / \AA^{2} \mathrm{ps}^{-1}$ & $D / \mathrm{cm}^{2} \mathrm{~s}^{-1}$ \\
\hline 1 (bulk-like water) & 0.8328 & $1.39 \times 10^{-5}$ \\
2 (outer membrane) & 0.2756 & $4.59 \times 10^{-6}$ \\
3 (inner membrane) & 0.1082 & $1.80 \times 10^{-6}$ \\
4 (inner membrane) & 0.0931 & $1.55 \times 10^{-6}$ \\
5 (outer membrane) & 0.3184 & $5.31 \times 10^{-6}$ \\
6 (bulk-like water) & 0.9068 & $1.51 \times 10^{-5}$ \\
\hline \hline
\end{tabular}


chosen water molecules and 475 choices of the time origin. From the slope of the mean-square displacement (not shown) in the range $10<t<100 \mathrm{ps}$, the diffusion constant is determined to be $D_{\mathrm{w} \text {, pure }}=2.33 \times 10^{-5} \mathrm{~cm}^{2} \mathrm{~s}^{-1}$ in agreement with the experimental value of $2.33 \times 10^{-5} \mathrm{~cm}^{2} \mathrm{~s}^{-1} \cdot 35$ However, this diffusion constant is larger than the diffusion constant in the membrane-associated bulk-like layer which is 1.45 $\times 10^{-5} \mathrm{~cm}^{2} \mathrm{~s}^{-1}$ averaged over the two bulk-like layers in Table III. The smaller diffusion constant reflects the influence of the lipids on the water dynamics in the bulk-like layers outside the membrane. In Ref. 14, it is found that the electrostatic potential from the charges in the headgroup of a DPPC bilayer membrane, which has the same headgroup as DMPC, extends out to $10-15 \AA$ from the surface of the membrane. This range of the electrostatic potential may explain the effect on the translational motion of the water molecules in the $\sim 10 \AA$ thick layer outside the membrane where the molecules have no direct contact with the lipid head groups. A similar explanation has been proposed for the fast rotational relaxation of the water molecules in a $\sim 10 \AA$ thick layer outside the DMPC membrane. ${ }^{12}$

\section{DIFFUSION OF SELECTED ATOMS IN DMPC}

We also investigated the diffusion of four selected atoms in the lipids for comparison with the diffusion of the water molecules in and near the membrane.

The displacement of an atom in a molecule may always be broken up into contributions from the center-of-mass and rigid rotational motion of the molecule and from intramolecular motions such as vibrations, librations, and conformational changes. ${ }^{36}$ The atomic displacement associated with a rotational motion and conformational changes will usually increase with the distance of the atom from the center of mass and may be significant in large molecules compared to the translational center-of-mass displacement. However, the rotational and intramolecular displacements are bounded so that after a sufficiently long time, the contributions from these motions to the mean-square displacement should be timeindependent. Thereafter, the time-dependence of the meansquare displacement is determined by the unbounded motions of the molecule; e. g., the lateral center-of-mass motion. Until the contributions from the bounded motions to the meansquare displacement have reached their limiting values, we will find that the diffusion constant (related to the slope in a plot of the mean-square displacement versus time) depends on the size of the time-window used in the calculations. Only for large enough time-windows will the diffusion constant be independent of the size of the time-window.

For the calculation of the mean-square displacement of the selected atoms, we have used a 2 ns time-window as in the calculation of the quasielastic scattering function described below. It is nearly the largest time-window we can use in the $3.5 \mathrm{~ns}$ time-bloc and still have satisfactory statistics at the longest times. A larger time-window may be used if several of the time block data files are concatenated but that was not needed here. The displacements were averaged over all atoms of a given kind and over about 700 different choices of time origin.
TABLE IV. The slope $\alpha$ of the linear part ( $1000<t<2000 \mathrm{ps})$ of the meansquare displacement curves and diffusion constants $D=\alpha / 6$ for the nitrogen, phosphorus, glycerol carbon, and carbonyl carbon atoms in the DMPC lipid molecule at $303 \mathrm{~K}$ and $1 \mathrm{~atm}$. The computation uses a $2 \mathrm{~ns}$ time-window and a 0.5 ps time resolution.

\begin{tabular}{lll}
\hline \hline Group & $\alpha / \AA^{2} \mathrm{ps}^{-1}$ & $D / \mathrm{cm}^{2} \mathrm{~s}^{-1}$ \\
\hline Nitrogen & $1.14 \times 10^{-2}$ & $1.90 \times 10^{-7}$ \\
Phosphorus & $7.45 \times 10^{-3}$ & $1.24 \times 10^{-7}$ \\
Carbonyl & $5.68 \times 10^{-3}$ & $9.47 \times 10^{-8}$ \\
Glycerol & $5.37 \times 10^{-3}$ & $8.94 \times 10^{-8}$ \\
\hline \hline
\end{tabular}

From a linear fit to the displacement curves (not shown) in the long-time range $1000<t<2000 \mathrm{ps}$, we found the slopes and diffusion constants compiled in Table IV. The different slopes or diffusion constants illustrate the point made above that contributions from bound motions to the meansquare displacement have not reached their limiting constant values within a 2 ns time-window. We can relate these differences in diffusion constants in Table IV to the average distance of the atoms from the center-of-mass. By averaging over all lipid molecules and time frames, we have determined the average distance of the selected atoms from the center-ofmass of the lipid molecules and found the following results: nitrogen, $9.44 \pm 1.17 \AA$; phosphorous, $7.11 \pm 0.78 \AA$; glycerol carbon atom, $3.72 \pm 1.04 \AA$; and carbonyl carbon atoms, $3.15 \pm 1.04 \AA$ and $3.34 \pm 1.12 \AA$.

We see that the mean-square displacement and diffusion constant are largest for the nitrogen atoms in the head group, because the amino group is furthest away from the center of mass located near the glycerol backbone. Therefore, the $\mathrm{N}$ atoms have some wagging motion in addition to a translational motion. The glycerol backbone carbon atom and carbonyl carbon atom have practically the same mean-square displacements and diffusion constants because they are close to each other and near the center-of-mass of the lipid. Their motion is likely dominated by the center-of-mass translational motion. The diffusion constant for the phosphorous atom is slightly larger being further away from the center of mass but closer than the nitrogen atom.

We have no estimate of the time it takes for the bounded rotational and intramolecular motions to reach a limiting constant contribution to the mean-square displacement. In principle, this time may be found by considering larger and larger time-windows until the diffusion constants are independent of the size of the time-window.

Another bounded motion in the bilayer membrane is the $z$-motion of the atoms and the lipid molecules' center-ofmass as illustrated by the number density distribution functions of the selected atoms in Fig. 2. It will be interesting to investigate whether the motion in the $z$-direction in a $2 \mathrm{~ns}$ time-window is a bounded or a free-translational diffusional motion. We have determined the 2D lateral $(x y)$ and 1D $(z)$ mean-square displacements for the selected atoms as plotted in Fig. 5. It is seen that the motion in the $z$ direction in a 2 ns time-window is an unbounded free-translational diffusion with a linear relationship between the mean-square displacement and time. From linear fits to the displacement 

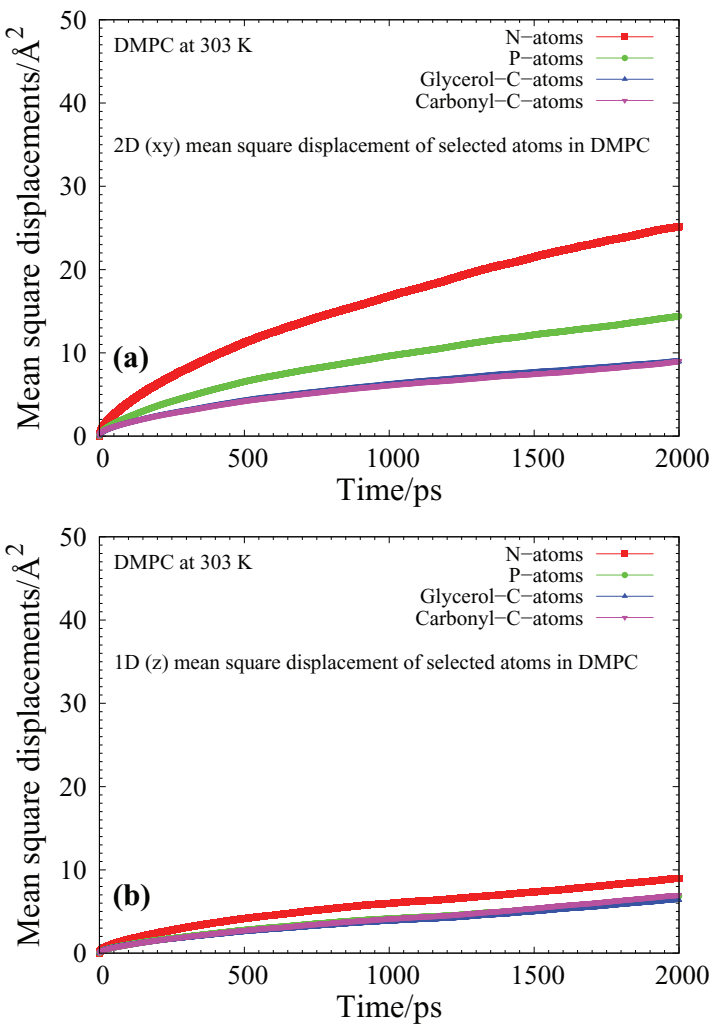

FIG. 5. (a) 2D (xy) mean-square displacements of the nitrogen, phosphorous, carbonyl carbon, and glycerol carbon atoms in the DMPC membrane at $303 \mathrm{~K}$ and $1 \mathrm{~atm}$ as function of time, using a 2 ns time-window and a 0.5 ps time-resolution. (b) $1 \mathrm{D}(z)$ mean-square displacements of the nitrogen, phosphorous, carbonyl carbon, and glycerol carbon atoms in the DMPC membrane at $303 \mathrm{~K}$ and $1 \mathrm{~atm}$ as function of time, using a 2 ns time-window and a $0.5 \mathrm{ps}$ time resolution.

curves in the time interval $1000<t<2000 \mathrm{ps}$, we have determined the $2 \mathrm{D}(x y)$ diffusion constants for lateral motion and the 1D $(z)$ diffusion constants for motion in the $z$ direction and compiled the results in Table $\mathrm{V}$ together with the 3D diffusion constant ( $x y z)$ from Table IV. The out-of-plane diffusion constants of atoms of the glycerol backbone (Table V) compare rather well with a neutron

TABLE V. The 2D (xy) diffusion constant for lateral motion, $D_{x y}$, and the 1D $(z)$ diffusion constant for motion in the $z$-direction, $D_{z}$. The diffusion constants are calculated from the slopes $\alpha$ of the mean-square displacement curves in Fig. 5 in the time range $1000-2000$ ps by the relation $D$ $=\alpha /(2 N D I M)$ where NDIM is the dimension. For the center-of-mass data we used the time range 500-1500 ps because the data showed evidence of statistical uncertainties at larger times. The computation uses a 2 ns timewindow and a $0.5 \mathrm{ps}$ time resolution. The $3 \mathrm{D}$ (xyz) diffusion constants for motion in all three Cartesian directions, $D_{x y z}$ from Table IV, have also been included for convenience.

\begin{tabular}{lccc}
\hline \hline Atom & $\begin{array}{c}3 \mathrm{D} \\
D_{x y z} / \mathrm{cm}^{2} \mathrm{~s}^{-1}\end{array}$ & $\begin{array}{c}2 \mathrm{D} \\
D_{x y} / \mathrm{cm}^{2} \mathrm{~s}^{-1}\end{array}$ & $\begin{array}{c}1 \mathrm{D} \\
D_{z} / \mathrm{cm}^{2} \mathrm{~s}^{-1}\end{array}$ \\
\hline Nitrogen & $1.90 \times 10^{-7}$ & $2.10 \times 10^{-7}$ & $1.51 \times 10^{-7}$ \\
Phosphorus & $1.24 \times 10^{-7}$ & $1.17 \times 10^{-7}$ & $1.39 \times 10^{-7}$ \\
Carbonyl & $9.47 \times 10^{-8}$ & $6.93 \times 10^{-8}$ & $1.47 \times 10^{-7}$ \\
Glycerol & $8.94 \times 10^{-8}$ & $6.70 \times 10^{-8}$ & $1.34 \times 10^{-7}$ \\
Center of mass & $9.76 \times 10^{-8}$ & $9.52 \times 10^{-8}$ & $1.02 \times 10^{-7}$ \\
\hline \hline
\end{tabular}

scattering measurement of the out-of-plane diffusion constant $\left(\sim 0.6 \times 10^{-7} \mathrm{~cm}^{2} \mathrm{~s}^{-1}\right){ }^{10}$ Because it is a bounded motion, some of the discrepancy with experiment may be due to differences in the time windows involved in the determination.

We note that the diffusion constants for motion in the $z$-direction are practically identical for all selected atoms in the lipid, so the differences in the 3D diffusion constants originate in differences in the lateral motion. The lateral diffusion constants are smaller than the diffusion constant for motion in the $z$-direction except for the nitrogen atoms. They increase as we get further away from the center-of-mass (near the glycerol backbone), which illustrates the importance of the contribution to the mean-square displacement from rotational motion. As a check of these results, we see that the 3D diffusion constant is equal to a weighted average of the 2D and 1D diffusion constants with weight factors 2 and 1 , respectively.

The distribution functions for the selected atoms in Fig. 2 represent a time-independent equilibrium distribution of atoms along $z$ obtained after simulations for 20-30 ns with the revised force field. Recall that these computations followed simulations for $60 \mathrm{~ns}$ with the original force field as discussed in Sec. II. The FWHM of the peaks of the distribution functions $(\sim 7-8 \AA$ ) may therefore be considered a measure of the bound on the motion in the $z$-direction. They may be used to estimate the length of time $t \sim F W H M^{2} /\left(2 D_{z}\right) \sim 20 \mathrm{~ns}$ for which the motion in the $z$-direction appears to be an unbounded diffusive motion. This result is consistent with our finding that the motion is unbounded in a 2 ns time-window [Fig. 5(b)].

We have also determined the 3D (xyz), 2D (xy), and 1D (z) center-of-mass diffusion constants for the lipid molecules with the results given in Table V. The motion in the $z$-direction also appears as unbounded with a non-zero diffusion constant in the $2 \mathrm{~ns}$ time-window. If we increase the size of the time-window, we should see the 1D (z) diffusion constant approach zero as the mean-square displacement in the $z$-direction reaches its time-independent limit. Note that the 2D (xy) lateral diffusion constant should be unchanged because the lateral motion is unbounded. The $2 \mathrm{D}(x y)$ center-ofmass lateral diffusion constant should therefore be the limiting value of the (xy) diffusion constants for all of the selected atoms in the lipid head groups, when the contributions from their bounded motions are time-independent.

In the mean-square displacement calculations for the center-of-mass motion, there was evidence of poor statistics at long times (not shown); so we have only used the time range 500-1500 ps in the linear fits to the curves. This uncertainty may explain the slightly larger diffusion constant for the center-of-mass motion compared to the diffusion constants for the glycerol and carbonyl carbon atoms, which, of course, is unphysical. They should be identical to the centerof-mass diffusion constant or slightly larger as they are close to the center of mass.

In summary, the mean-square displacement of the atoms in the relatively small water molecules will be dominated by the translational center-of-mass displacement after a very short time, whereas that may not be the case for the atoms 
in the much larger lipid molecule. The mean-square displacement results from a combination of a center-of-mass, rotational, and conformational motion of the molecule. We do not have an estimate of the time necessary for the lateral translational displacement to dominate the intramolecular bounded displacements but have estimated that it takes $\sim 20 \mathrm{~ns}$ for the bounded translational displacement along $z$ to reach its timeindependent limiting value.

\section{BINDING OF WATER TO SELECTED ATOMS IN DMPC}

In this section, we describe an analysis of the binding of water molecules to selected atoms in the DMPC molecule. It involves two steps. First, we determine the distribution of water molecules as a function of the distance from the selected atoms in the lipids and identify the range of the solvation shells. Then, the kinetics of the water molecules in the shells is analyzed by determining their rate of disappearance from the solvation shells. Water molecules that are loosely bound to the atoms will disappear from the shell at a faster rate than ones more strongly bound, so their rate of disappearance may be used as a relative measure of their binding strength.

\section{A. Distribution of water molecules around selected atoms}

The distribution of water molecules around the selected atoms in the lipid is most likely anisotropic, in contrast to the distribution of water molecules around a single ion in solution, because each atom in the membrane is bound to other atoms or a group of atoms that may block the water molecules. We have not analyzed this anisotropy, so our results represent an average, isotropic distribution. The space around the individual atoms has been divided into spherical shells of thickness $d R=0.1 \AA$, and the number of water molecules within each shell has been counted and converted to a number density by division by the shell volume. The results are averaged over all selected atoms and 900 choices of time origins and presented in Fig. 6.

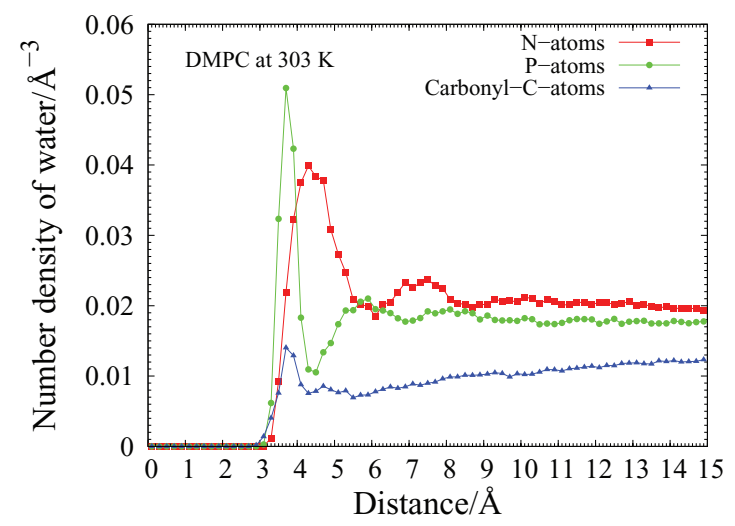

FIG. 6. The isotropic number density of water molecules around specified atoms in the DMPC lipid head groups at $303 \mathrm{~K}$ and $1 \mathrm{~atm}$ as a function of the distance from the atoms.
From the figure, we see that the best defined first solvation shell appears to be the one around the phosphorous atoms given by radii in the range $\sim 3.0-4.0 \AA$. The peak position at $\sim 3.7 \AA$ shows that the water molecules are a little closer to the phosphorous atoms than to the nitrogen atoms with their broader first solvation shell defined by radii in the range 3.0$6.0 \AA$ and a peak position at $\sim 4.5 \AA$. Because the phosphorous peak position is at a larger distance than the $\mathrm{P}-\mathrm{O}$ distance in the phosphate group $\sim 1.5-1.7 \AA$, the water molecules appear to be packed around the P-O group as a whole. The broader first-shell peak for the nitrogen atoms may reflect the presence of the three methyl groups attached to them to form the amino group. The distance from the nitrogen atom to the hydrogen atoms in the attached methyl groups is $\sim 2.1-2.3 \AA$, which shows that the water molecules are packed around the $\mathrm{N}-3\left(\mathrm{CH}_{3}\right)$ group as a whole. The peak position for the carbonyl carbon atoms is almost the same as for the phosphorous atoms, and the first solvation shell is given by radii in the range $\sim 3.0-4.2 \AA$. The glycerol carbon atoms have not been included in the analysis because we have focused on the polar groups in the head group as being most important for the binding of water.

There is a clear second solvation shell around the nitrogen and phosphorous atoms but not around the carbonyl carbon atoms. At larger distances, the distribution functions level out to an almost constant number density, somewhat lower for the carbonyl carbon atoms than for the nitrogen and phosphorous atoms. These levels correlate well with the number density curves in Fig. 2.

\section{B. Kinetic analysis of water molecules around select atoms}

If the water molecules in a solvation shell around an atom are rigidly bound to the atom, they will follow its motion and be found in the solvation shell at all times just as one atom in a dimer follows the other. Therefore, we may estimate the relative strength of the binding by determining the rate at which water molecules leave a given solvation shell around the atoms. Tightly bound water molecules will on the average stay for a longer time in the solvation shell than ones more loosely bound.

We have only considered the rate by which water molecules leave the first solvation shell because the analysis of second solvation shell molecules is more complex. In leaving the second solvation shell, molecules may enter the first solvation shell and therefore not leave the vicinity of the atom. Also, the second solvation shell is not as well defined as the first solvation shell.

The calculations of the water kinetics are done in the following way. For a chosen time origin, we tag all water molecules found in the first solvation shell defined by the two radii $R_{\min }$ and $R_{\max }$ around the selected atoms in the lipid head groups. We then follow the motion of these atoms, determine the distances to the tagged water molecules around them, and count the number of tagged water molecules in the first solvation shell as a function of time. The results are averaged over all atoms of a given kind and over 900 different choices of time origins. 


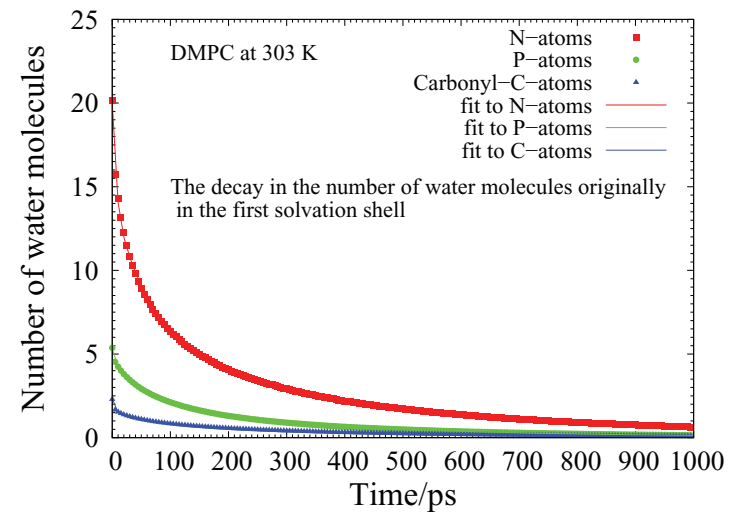

FIG. 7. The decay in the number of tagged water molecules as a function of time in the first solvation shell around the nitrogen, phosphorous, and carbonyl carbon atoms, respectively, in a DMPC lipid bilayer membrane at $303 \mathrm{~K}$ and $1 \mathrm{~atm}$. The fitting parameters for the decay curves are found in Table VI where the definitions of the first shells also are given. The data were recorded with a 5 ps time-resolution.

In Fig. 7, we have shown the decay in the number of water molecules in the first shell around the nitrogen, phosphorous, and carbonyl carbon atoms as function of time. We have used first order kinetics

$$
\frac{d n_{\mathrm{w}}(t)}{d t}=-k n_{\mathrm{w}} \Rightarrow n_{\mathrm{w}}(t)=n_{\mathrm{w}}(0) \exp (-k t)
$$

to parameterize the decay curves. $k$ is the rate constant for the decay, and $n_{\mathrm{w}}(t)$ is the number of tagged water molecules at time $t$ in the given shell. It is easy to prove that the reciprocal rate constant $1 / k=\tau_{\text {res }}$ is equal to the average residence time $\tau_{\text {res }}$ of the tagged water molecules in the shell. From the decay curves, it is evident that they cannot be fit by a single exponential. Therefore, we have fitted them to several exponentials using

$$
n_{\mathrm{w}}(t)=\sum_{i=1}^{\max } a_{i} \exp \left(-k_{i} t\right)
$$

We used three exponentials $(\max =3)$ to obtain a satisfactory fit to the data over the entire time range. Three exponentials also turned out to be the maximum number which yielded physically reasonable parameters. The fitting of three exponentials to a decay curve is a delicate process; and the results may be sensitive to the time-window used for the fit as pointed out, for example, in Ref. 17. We have used a large time-window $(\sim 1 \mathrm{~ns})$ in the fit, which may give more reliable results especially for the component with the smallest rate constant.

Table VI contains the results of the kinetic studies and shows that the water molecules around selected atoms in the DMPC molecule may be divided into three categories according to the average residence times or strength of their binding. The residence times change by two orders of magnitude from $\sim 5 \mathrm{ps}$ for the loosely bound water molecules to $\sim 70 \mathrm{ps}$ for water molecules bound with an intermediate strength and then to $\sim 400 \mathrm{ps}$ for the most strongly bound molecules. There are some differences in the distribution (the $a_{i}$ parameters) of the molecules over these different categories for the three atoms. For both the nitrogen and phosphorous atoms, most
TABLE VI. The fitting parameters $a_{i}, k_{i}$ of Eq. (2) characterizing the timedecay for the number of tagged water molecules in the first solvation shell around the nitrogen, phosphorous, and carbonyl carbon atoms in the DMPC lipid head group in a bilayer membrane at $303 \mathrm{~K}$ and $1 \mathrm{~atm}$. The first solvation shell for the nitrogen atoms is defined by the distance $R$ in the range $3.0<R$ $<6.0 \AA$, for the phosphorous atoms by the range $3.0<R<4.5 \AA$, and for the carbonyl carbon atoms by the range $3.0<R<4.2 \AA$. The definition of the solvation shells is based on the number density profiles in Fig. 6. The fraction $100 a_{i} / \sum_{j} a_{j}$ (in $\%$ ) of water molecules with different residence times $\tau_{i}$ and the number of molecules initially in the solvation shell are also given. The fit was done using data in a $1 \mathrm{~ns}$ time-window with a 5 ps time resolution.

\begin{tabular}{lcccc}
\hline \hline Parameters & Nitrogen & Phosphorous & Carbonyl group & Average \\
\hline Molecules at start & 20.15 & 5.37 & 2.29 & $\ldots$ \\
$a_{1}$ molecules & 5.79 & 0.95 & 0.75 & $\ldots$ \\
$100 a_{1} / \sum_{j} a_{j}(\%)$ & 28.9 & 17.8 & 30.8 & 25.8 \\
$k_{1}^{-1}=\tau_{1} /$ ps & 6.25 & 5.73 & 3.23 & 5.1 \\
$a_{2}$ molecules & 8.57 & 2.52 & 0.79 & $\ldots$ \\
$100 a_{2} / \sum_{j} a_{j}(\%)$ & 42.8 & 47.1 & 34.5 & 41.4 \\
$k_{2}^{-1}=\tau_{2} /$ ps & 66.7 & 76.9 & 74.1 & 72.6 \\
$a_{3}$ molecules & 5.66 & 1.88 & 0.80 & $\ldots$ \\
$100 a_{3} / \sum_{j} a_{j}(\%)$ & 28.3 & 35.1 & 34.7 & 32.7 \\
$k_{3}^{-1}=\tau_{3} / \mathrm{ps}$ & 434.7 & 375.9 & 429.2 & 413.33 \\
\hline \hline
\end{tabular}

of the water molecules belong to the category bound with an intermediate strength (category 2). However, the fraction of molecules loosely bound to the phosphorous atoms is smaller than for the nitrogen atoms. This result appears to be consistent with the narrower width of the 1st solvation shell for phosphorous. There is almost an even distribution over categories for the carbonyl group. If we arbitrarily assume that the water molecules with the largest residence times in the kinetic analysis follow the lipid molecules, we find that there are $\sim 8.4$ tightly bound water molecules per lipid molecule. This estimate appears to be an upper bound because with an average residence time of $\sim 0.4 \mathrm{~ns}$, it is unlikely that all of these molecules can follow the lipid molecules which move on a nanosecond time scale.

\section{QUASIELASTIC NEUTRON SCATTERING FUNCTIONS}

We have calculated both the quasielastic incoherent intermediate scattering function $F_{\mathrm{s}}(q, t)$ and the quasielastic incoherent scattering function $S_{\mathrm{s}}(q, \omega)$ for water and the selected atoms in the lipid head group in order to compare with the experimental quasielastic neutron scattering results. by $^{34}$

The incoherent intermediate scattering function is given

$$
F_{\mathrm{s}}(q, t)=\frac{1}{N} \sum_{i} b_{i}^{2}\left\langle\exp \left(i \mathbf{q} \cdot\left(\mathbf{r}_{i}(t)-\mathbf{r}_{i}\left(t_{0}\right)\right)\right)\right\rangle,
$$

where the brackets symbolize an ensemble average of the enclosed term. The ensemble average includes an average over orientations of the difference vector $\mathbf{r}_{i}(t)-\mathbf{r}_{i}\left(t_{0}\right)$ between positions of atom $i$ at time $t$ and time $t_{0}$ with respect to the scattering vector $\mathbf{q}$ which is in the plane of the membrane. The averaging over orientations is often done analytically assuming an isotropic orientation of the difference vector with respect to the scattering vector in either two- or three dimensions. 
We have used two averages, a 2D cylindrical average for the selected atoms in the lipids because they do not "tumble" around but have a permanent preferred orientation with respect to the scattering vector and a 3D spherical average for the water molecules that essentially "tumble" around in all orientations as in bulk water.

The cylindrical average results in the zero order cylindrical Bessel function $J_{0}$ of the first kind ${ }^{37}$ so that the intermediate scattering functions for the selected atoms in the lipid head groups are calculated from the expression

\section{Cylindrical average:}

$$
F_{\mathrm{s}}(q, t)=\frac{1}{N} \sum_{i}\left\langle b_{i}^{2} J_{0}\left(\left|\mathbf{q} \| \mathbf{r}_{i}(t)-\mathbf{r}_{i}\left(t_{0}\right)\right|\right)\right\rangle,
$$

while the spherical average gives the zero order spherical Bessel function $j_{0}$ of the first kind ${ }^{37}$ and the intermediate scattering function

Spherical average:

$$
\begin{aligned}
F_{\mathrm{s}}(q, t) & =\frac{1}{N} \sum_{i}\left\langle b_{i}^{2} j_{0}\left(\left|\mathbf{q} \| \mathbf{r}_{i}(t)-\mathbf{r}_{i}\left(t_{0}\right)\right|\right)\right\rangle \\
& =\frac{1}{N} \sum_{i}\left\langle b_{i}^{2} \frac{\sin \left(\left|\mathbf{q} \| \mathbf{r}_{i}(t)-\mathbf{r}_{i}\left(t_{0}\right)\right|\right)}{\left|\mathbf{q} \| \mathbf{r}_{i}(t)-\mathbf{r}_{i}\left(t_{0}\right)\right|}\right\rangle .
\end{aligned}
$$

The brackets in the expressions refer to an ensemble average of the term inside them. It is determined as an average of contributions from all atoms $i$ of the given type and over a series of different choices of the initial time $t_{0} . b_{i}$ is the atomic scattering length which arbitrarily has been set to one in the calculations because we do not compare scattering intensities for different atoms. The incoherent scattering function $S_{\mathrm{s}}(q, \omega)$ is determined as the time-Fourier transform of the intermediate scattering function

$$
S_{\mathrm{s}}(q, \omega)=\int_{0}^{t_{\mathrm{window}}} d t \exp (i \omega t) F_{\mathrm{s}}(q, t),
$$

which is proportional to the quasielastic intensity in the scattering experiments.

If the intermediate scattering function has not decayed to zero within the time window, there will be an elastic scattering in addition to a quasielastic scattering with a strength proportional to the value of the intermediate scattering function at time $t=t_{\text {window }}$. Before determining the time-Fourier transform of such a function, it is important to eliminate the elastic contribution to the scattering by subtracting the value of the intermediate scattering function at $t=t_{\text {window }} \cdot{ }^{38}$ Otherwise, the incoherent quasielastic scattering function will be obscured by the contribution from the elastic scattering which will not be confined to the region near $\omega=0$ because of truncation errors in doing the Fourier transform.

The incoherent scattering functions have been fitted to Lorentzians according to

$$
S_{\mathrm{s}}(q, \omega)=\frac{1}{\pi} \sum_{i=1}^{n \max } a_{i} \frac{\Gamma_{i}(q)}{\omega^{2}+\Gamma_{i}(q)^{2}},
$$

where $\omega=2 \pi \nu$ is the angular frequency, $a_{i}$ is proportional to the intensity of the quasielastic scattering, and $\Gamma_{i}(q)$ the half- width-at-half maximum (HWHM) of the Lorentzian, which may be a function of the wave vector transfer $q$ depending on the kind of diffusive motion. The Lorentzian line shape is chosen in the fits, because it can be shown ${ }^{34,39}$ that the scattering function is a Lorentzian for simple types of diffusive motions such as random translational diffusion and random isotropic rotational diffusion.

The $q$ dependence of the HWHM may be used to infer the type of diffusive motion involved. Thus, for random translational diffusive motion, the HWHM is proportional to $q^{2}$, that is,

$$
\Gamma(q)=D q^{2},
$$

where the coefficient of proportionality $D$ is the translational diffusion constant. For random isotropic rotational motion, the HWHM is independent of $q$. For more complicated diffusive motions, no simple theoretical expression exists for the $q$-dependence of the HWHM. However, some $q$ dependence of $\Gamma(q)$ may indicate that translational diffusive motion is involved, while little or no $q$ dependence may indicate that rotational diffusive motion dominates the scattering.

Equation (7) raises the question of how many Lorentzians should be used in a fit? The upper limit is reached when the addition of one more Lorentzian in the fit does not allow their parameters to be determined unambiguously. Another criterion is to check by direct inspection the fit to the data. For the water molecules, for example, it was obvious that a single Lorentzian fit was inadequate (not shown). The fit simply had a wrong "shape" at small frequencies which could be corrected with a two-Lorentzian fit. A third criterion for how many Lorentzians to use in a fit is to look at the intensities. If the intensity of a Lorentzian is two or more orders of magnitude less than the intensities of the other Lorentzians, its contribution to the incoherent scattering function is only marginal. In this case, it becomes problematic whether its parameters are physically significant especially when the shape of the fit is correct before the addition of the extra Lorentzian.

\section{A. Results}

We have determined the scattering functions for the atoms at two of the wave vectors, $q=0.5 \AA^{-1}$ and $0.9 \AA^{-1}$ investigated in the experiments. ${ }^{20}$ As an example, we show the intermediate scattering function for water at $q=0.5 \AA^{-1}$ in Fig. 8(a) and the scattering function in Fig. 8(b). The fitting parameters $a_{i}, \Gamma_{i}$ in Eq. (7) for all atoms considered are collected in Tables VII and VIII. They show that a twoLorentzian fit was required in the fitting of the scattering functions so that the motion of the atoms is on at least two time scales, a slow one $\left(\Gamma_{1}\right)$ and a fast one $\left(\Gamma_{2}\right)$.

For the phosphorous, glycerol carbon, and carbonyl carbon atoms, we note that there is little variation among the three atoms in their slow and fast time scales, respectively, at both wave vectors. This behavior is expected because the atoms are close to each other near the glycerol backbone of the lipid. Both time scales for the nitrogen atoms are slightly larger than those for the other atoms probably because the atoms are located at the end of the head group further away from the glycerol backbone, allowing more flexibility and 

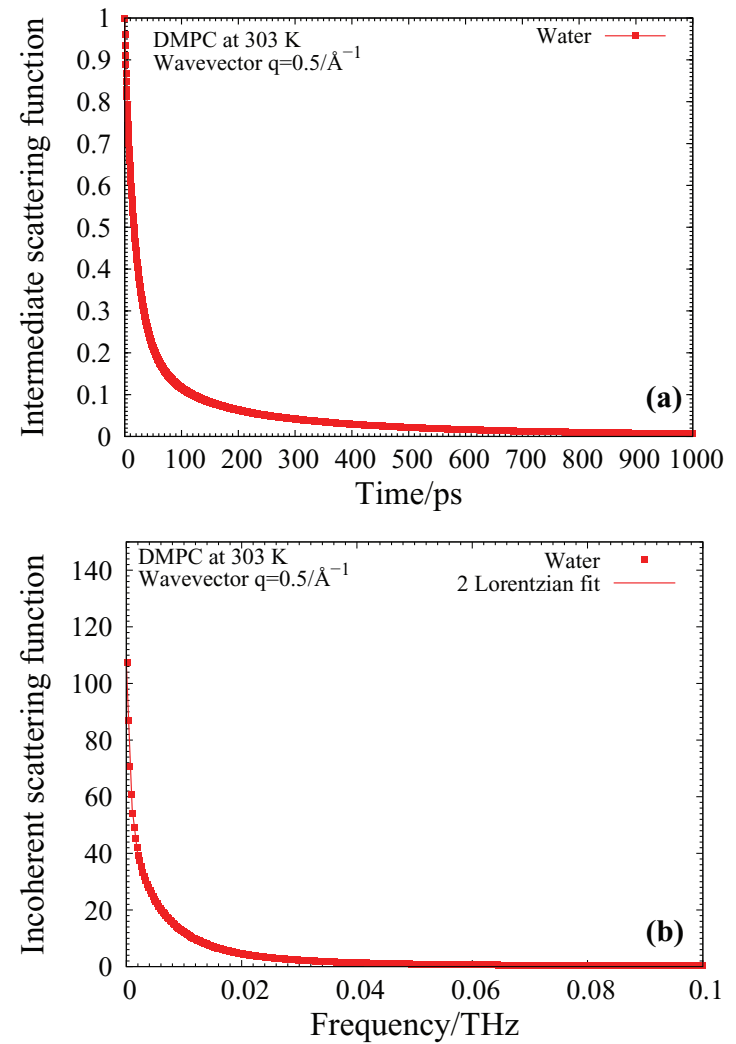

FIG. 8. (a) The intermediate scattering function for the water molecules in DMPC at $303 \mathrm{~K}$ and $1 \mathrm{~atm}$ for wave vector $q=0.5 \AA^{-1}$. (b) The incoherent scattering function at $q=0.5 \AA^{-1}$ and a two-Lorentzian fit. Note that the abscissa in the plot is the frequency $v$ and not the angular frequency $\omega$. The HWHM in the plot is multiplied by $2 \pi$ to obtain the $\Gamma_{i}$ in Eq. (7).

motion of the group. The fast time scale for water $\left(\Gamma_{2}\right)$ is much faster than for the atoms in the lipid, while its slow time scale $\left(\Gamma_{1}\right)$ is intermediate between their fast and slow time scales.

We have tried to identify the nature of the motion associated with the slow $\left(\Gamma_{1}\right)$ and fast $\left(\Gamma_{2}\right)$ components by considering the $q$-dependence of $\Gamma_{1}$ and $\Gamma_{2}$. From Tables VII and VIII, it is evident that all $\Gamma$ depend on $q$, indicating that none of the components represent a "pure" random rotational motion. To test whether the components may be associated with "pure" random translational motion, we have used Eq. (8) and calculated the ratio $\Gamma / q^{2}$ at the two wave vectors. It should be independent of $q$ for "pure" translational motion. In
Table IX, we have summarized the differences in $\Gamma / q^{2}$ at the two wave vectors. It shows that there is a considerable deviation from linearity for the selected lipid atoms which implies that they do not undergo "pure" translational motion at the given length-scale $(2 \pi / q)$ but rather a more complex motion. In contrast, the fast component for water $\left(\Gamma_{2}\right)$ seems to be dominated by translational motion.

When we look at the slow component of water $\left(\Gamma_{1}\right)$, we note that it is intermediate between the values for the slow and fast components of the selected atoms. This behavior suggests that some of the water molecules may move on a similar time scale as the selected atoms. Indeed, if we had had the sensitivity, we might have been able to resolve the narrow peak $\left(\Gamma_{1}\right)$ for the water scattering into two peaks corresponding to two components having HWHMs similar to the two widths found for the selected atoms. Without this possibility, our fit results in an intermediate width for the water molecules. We also note that the $q$-dependence of the slow component of water differs greatly from that of the fast component as evidenced by Table IX. Because the motion of some water molecules is much slower than others but within the time scale for the selected atoms, it is natural to suggest that these molecules are strongly bound to the lipid molecules and follow their motion.

We have tried to identify the kind of motion dominating the two components for the selected lipid atoms. This identification has been made by assuming that they represent translational motion. We then compare the resulting translational diffusion constants with the mean-square displacement results to either confirm or falsify the assumption. In order to extract a translational diffusion constant from the width of the scattering function, the limit $q \rightarrow 0$ (infinite length scale) in Eq. (8) needs to be taken. This limit can be determined if a series of results at small wave vectors are available. Here we only have results at two wave vectors for which there is a considerable deviation from linearity. Therefore, the best way to extract a diffusion constant will be to use the results for the smallest wave vector, here $q=0.5 \AA^{-1}$. The results are shown in Table X. We see that the $D_{1}$-values for the selected atoms are closest to the $D$-values from the mean-square displacement calculations; we conclude that the $\Gamma_{1}$ component seems to be dominated by translational motion, while the faster $\Gamma_{2}$ component is dominated by non-translational motions such as vibrations and librations. This identification is just opposite that for water where the fast component was associated with translational motion. For completeness, we have also included

TABLE VII. The fitting parameters, $a_{i}, \Gamma_{i}$, for the two-Lorentzian fits to the simulated incoherent scattering functions for selected atoms in the lipid head groups and water molecules in the DMPC lipid bilayer membrane at $303 \mathrm{~K}$ and $1 \mathrm{~atm}$. The analysis is performed at a wave vector transfer $q=0.5 \AA^{-1}$. The time window in the calculations is $2 \mathrm{~ns}$ and the time resolution is $0.5 \mathrm{ps}$, corresponding to an energy resolution of $\sim 2.1 \mu \mathrm{eV}$ and a dynamic range of $\sim 4.13 \mathrm{meV}$. The "rms" entry in the table is the root-mean-square deviation in the fit.

\begin{tabular}{llllll}
\hline \hline Parameters & Nitrogen & Phosphorous & Glycerol-C & Carbonyl-C & Water \\
\hline$a_{1}$ & $5.22 \times 10^{-1}$ & $5.27 \times 10^{-1}$ & $4.48 \times 10^{-1}$ & $4.51 \times 10^{-1}$ & $1.56 \times 10^{-1}$ \\
$\Gamma_{1} / \mu \mathrm{eV}$ & $7.10 \times 10^{-1}$ & $5.4 \times 10^{-1}$ & $5.30 \times 10^{-1}$ & $5.3 \times 10^{-1}$ & 2.79 \\
$a_{2}$ & $3.22 \times 10^{-1}$ & $2.27 \times 10^{-1}$ & $1.66 \times 10^{-1}$ & $1.68 \times 10^{-1}$ & $7.85 \times 10^{-1}$ \\
$\Gamma_{2} / \mu \mathrm{eV}$ & 4.09 & 3.52 & 3.16 & 3.21 & 30.60 \\
$\mathrm{rms}$ & 0.24 & 0.12 & 0.11 & 0.11 & 0.14 \\
\hline \hline
\end{tabular}


TABLE VIII. The fitting parameters, $a_{i}, \Gamma_{i}$, for the two-Lorentzian fits to the simulated incoherent scattering functions for different atoms and molecules in the DMPC lipid membrane at $303 \mathrm{~K}$ and $1 \mathrm{~atm}$. The analysis is performed at a wave vector transfer $q=0.9 \AA^{-1}$. The time window in the fits is $2 \mathrm{~ns}$ and the time resolution is $0.5 \mathrm{ps}$, corresponding to an energy resolution of $2.1 \mu \mathrm{eV}$ and a dynamical range of $1 \mathrm{THz}=4.13 \mathrm{meV}$. The "rms" entry in the table is the root mean square deviation in the fit.

\begin{tabular}{llllll}
\hline \hline Parameters & Nitrogen & Phosphorous & Glycerol-C & Carbonyl-C & Water \\
\hline$a_{1}$ & $5.08 \times 10^{-1}$ & $5.46 \times 10^{-1}$ & $6.12 \times 10^{-1}$ & $6.31 \times 10^{-1}$ & $1.28 \times 10^{-1}$ \\
$\Gamma_{1} / \mu \mathrm{eV}$ & 2.55 & 1.48 & 1.28 & 1.42 & 6.33 \\
$a_{2}$ & $4.10 \times 10^{-1}$ & $3.82 \times 10^{-1}$ & $2.76 \times 10^{-1}$ & $2.50 \times 10^{-1}$ & $7.74 \times 10^{-1}$ \\
$\Gamma_{2} / \mu \mathrm{eV}$ & 17.28 & 8.14 & 7.78 & 9.97 & 97.19 \\
$\mathrm{rms}$ & 0.24 & 0.26 & 0.26 & 0.22 & 0.14 \\
\hline \hline
\end{tabular}

the simulated and experimental bulk water diffusion constants in Table X. The agreement between the simulated diffusion constant for all water molecules and the experimental value ${ }^{20}$ is very good. Also, we see that the agreement between the two ways of determining the diffusion constant for bulk water in a simulation is reasonable.

From the intensities of the fast and slow components $\left(a_{1}, a_{2}\right)$ for water in Tables VII and VIII we see from the $q=0.5 \AA^{-1}$ data that $17 \%$ of the water molecules or 6.4 water molecules/lipid are strongly bound to the lipid and from the $q=0.9 \AA^{-1}$ data that $14 \%$ or 5.5 water molecules/lipid are strongly bound. These numbers are somewhat less than the $\sim 8$ water molecules/lipid as estimated from the kinetic studies. Considering that the longest average residence time is $\sim 0.4 \mathrm{~ns}$, it is clear that only some fraction of the 8 water molecules will have residence times on the order of the 1-2 ns time scale for the motion of the selected atoms. Therefore, 8 water molecules/lipid must be an upper limit to the number of bound water molecules as is consistent with the scattering results.

In summary, the simulations of the scattering functions at the given temperature have shown that one may identify two types of water molecules: (1) those that are strongly bound to atoms in the lipid ( $\sim 6$ water molecules/lipid) and move on the same time scale as atoms in the lipid; and (2) those that move faster but still slower than in bulk and which are more loosely bound to the lipids. It should be noted that the diffusion constant for the second type of water will depend on the amount of water in the system. In the simulations, we had a $\sim 10 \AA$ water layer outside the membrane in which the dynamics was still affected by the membrane. But, if we increase the thickness of that water layer, the influence of the membrane on the dynamics of water further away from the membrane surface will diminish so that the diffusion constant will approach that of bulk water.

TABLE IX. Test of the linear relationship between the width $\Gamma$ and the square of the wave vector transfer $q^{2}$ (Eq. (8)) at $q=0.5 \AA^{-1}$ and $q$ $=0.9 \AA^{-1}$. The relative deviation $\left(\Gamma / 0.9^{2}-\Gamma / 0.5^{2}\right) /\left(\Gamma / 0.5^{2}\right)$ in percent is given for each of the two $\Gamma$.

\begin{tabular}{lccccr}
\hline \hline Width & Nitrogen & Phosphorous & Glycerol-C & Carbonyl-C & Water \\
\hline$\Gamma_{1}$ & 10.0 & -15.3 & -25.5 & -17.5 & -30.6 \\
$\Gamma_{2}$ & 30.4 & -28.6 & -24.0 & -4.1 & -2.2 \\
\hline \hline
\end{tabular}

\section{COMPARISON WITH EXPERIMENTS}

The experiments ${ }^{20}$ were done on state-of-the-art backscattering spectrometers: the High-Flux Backscattering Spectrometer (HFBS) at the NIST Center for Neutron Research $^{40}$ and BASIS at the Spallation Neutron Source (SNS), Oak Ridge National Laboratory. ${ }^{41}$ The large dynamical range of BASIS allowed us to resolve two diffusive processes corresponding to the two Lorentzians in the fit to the quasielastic scattering intensity, a broad component, which has a $q^{2}$ dependence at low $q$ characteristic of translational diffusion and a narrow component with only a weak $q$ dependence. The broad component observed represents relatively fast moving water molecules which above the freezing transition at $268 \mathrm{~K}$ (lower than $273 \mathrm{~K}$ due to hysteresis) are referred to as "bulk-like" water located outside the membrane. Below $268 \mathrm{~K}$, the broad component represents "confined" water located in and immediately outside the membrane.

Because the simulations have only been done at one temperature, $303 \mathrm{~K}$, in contrast to the experiments that are conducted over a wide range of temperatures, we are limited in

TABLE X. Comparison of diffusion constants for water molecules and selected atoms in the DMPC lipid bilayer membrane at $303 \mathrm{~K}$ and $1 \mathrm{~atm}$ obtained from simulations using mean-square displacements and quasielastic incoherent neutron scattering. Layers 1, 2, 3 refer to the diffusion constants for water in the bulk-like, outer membrane, and inner membrane layers, respectively, averaged over the two leaflets. The simulation results for bulk water and the measurements on single-supported DMPC membranes (Ref. 20) are also included.

\begin{tabular}{|c|c|c|c|}
\hline \multirow[t]{2}{*}{$\begin{array}{l}\text { Atom } \\
\text { type }\end{array}$} & \multirow{2}{*}{$\begin{array}{c}\begin{array}{c}\text { Mean sq. } \\
\text { displ. }\end{array} \\
D / \mathrm{cm}^{2} \mathrm{~s}^{-1}\end{array}$} & \multicolumn{2}{|c|}{$\begin{array}{l}\text { Incoherent quasielastic scattering } \\
\text { Wave vector } q=0.5 \AA^{-1}\end{array}$} \\
\hline & & $D_{1} / \mathrm{cm}^{2} \mathrm{~s}^{-1}$ & $D_{2} / \mathrm{cm}^{2} \mathrm{~s}^{-1}$ \\
\hline $\mathrm{N}$ & $1.90 \times 10^{-7}$ & $4.32 \times 10^{-7}$ & $2.49 \times 10^{-6}$ \\
\hline $\mathrm{P}$ & $1.24 \times 10^{-7}$ & $3.28 \times 10^{-7}$ & $2.14 \times 10^{-6}$ \\
\hline Glycerol C & $9.47 \times 10^{-8}$ & $3.22 \times 10^{-7}$ & $1.92 \times 10^{-6}$ \\
\hline Carbonyl C & $8.94 \times 10^{-8}$ & $3.22 \times 10^{-7}$ & $1.95 \times 10^{-6}$ \\
\hline Water (all) & $1.06 \times 10^{-5}$ & $1.70 \times 10^{-6}$ & $1.86 \times 10^{-5}$ \\
\hline Layer 1 & $1.45 \times 10^{-5}$ & $\ldots$ & $\ldots$ \\
\hline Layer 2 & $4.95 \times 10^{-6}$ & $\ldots$ & $\ldots$ \\
\hline Layer 3 & $1.68 \times 10^{-6}$ & $\ldots$ & . \\
\hline $\begin{array}{l}\text { Bulk water } \\
\text { (model) }\end{array}$ & $2.33 \times 10^{-5}$ & $\ldots$ & $2.71 \times 10^{-5}$ \\
\hline Expt. $^{\mathrm{a}}$ (Ref. 20) & $\ldots$ & $\ldots$ & $1.91 \times 10^{-5}$ \\
\hline
\end{tabular}


the comparison between the two that can be made. In particular, direct comparison of the "confined water" inferred experimentally with the simulation results is impossible, because the former can only be isolated experimentally at temperatures below $268 \mathrm{~K}$. Thus, we can only compare the HWHM and diffusion constant for the "bulk-like" water in the experiments with the simulations. For the broad component at $303 \mathrm{~K}$, the experiment gave a HWHM $\sim 35 \mu \mathrm{eV}$ at $q=0.5 \AA^{-1}$ and $\sim 90 \mu \mathrm{eV}$ at $q=0.9 \AA^{-1}$, which compare rather well with the simulation results, a HWHM $\sim 31 \mu \mathrm{eV}$ at $q=0.5 \AA^{-1}$ and $\sim 97 \mu \mathrm{eV}$ at $q=0.9 \AA^{-1}$ as listed in Tables VII and VIII.

The narrow component in the scattering function was determined in the experiments at larger wave vector transfers of $1.4,1.5$, and $1.7 \AA^{-1}$, so a direct comparison with the simulation results at smaller $q$ is impossible. The larger wave vector transfers were used to increase sensitivity to the narrow component. At these large values of $q$, the broad component became so wide relative to the dynamic range of the spectrometer that it became indistinguishable from the background intensity.

In the experiment, the HWHM of the narrow component $(\sim 2.5 \mu \mathrm{eV}$ at $303 \mathrm{~K})$, depends only weakly on $q$ which indicates that rotational diffusion may be involved as well as conformational changes of the aliphatic tails. From both quasielastic measurements and MD simulations, we have found evidence of similar motions in solid bulk alkane particles $^{42}$ and in monolayer alkane films. ${ }^{38,43}$ The experimentally inferred HWHM of the narrow component is comparable to the narrow components $\left(\Gamma_{1}\right)$ in Tables VII and VIII.

From an intensity analysis of the scattering in the experiments, it was concluded that $\sim 7-10$ water molecules/lipid contribute to the intensity of the narrow component and therefore move on the same time scale as the $\mathrm{H}$ atoms within the lipid molecules, implying that they are strongly bound to the head groups. A similar analysis of the simulation data in both Tables VII and VIII shows that $\sim 6$ water molecules/lipid move on the same time scale as the selected atoms and hence as the $\mathrm{H}$ atoms in the head groups. These values deduced from the simulations are in fair agreement with the number of strongly bound water molecules inferred experimentally. Due to possible differences in structure between the singlesupported membrane in the experiment and the freestanding membrane in the simulations, some caution is needed in comparing these values for the number of bound water molecules per lipid head group.

\section{CONCLUSIONS}

The number density distribution function in the $z-$ direction perpendicular to the membrane surface for the selected atoms in the DMPC bilayer membrane (nitrogen, phosphorous, glycerol carbon, carbonyl carbon, and methyl group carbon at the end of the aliphatic chains) is rather broad. The FWHM $\sim 7-8 \AA$ corresponds to a root-mean-square deviation of $\sim 3.2 \AA$.

The calculated density profile of water molecules in the $z$-direction showed that they penetrate into the region of the head groups in the lipid molecules. Water molecules extend down to the carbonyl groups connecting the glycerol back- bone and the aliphatic chains. In the hydrophobic region of the aliphatic chains, no water molecules were found on the nanosecond time scale considered in this study.

Three layers have been defined in the $z$-direction in each leaflet of the membrane: a bulk-like water layer just outside the membrane which has the same density as bulk water, an outer membrane layer around the nitrogen and phosphorus atoms in the head group, and an inner layer including the glycerol backbone, carbonyl groups, and aliphatic chains. Our previous quasielastic neutron scattering experiments could not distinguish between these "outer" and "inner" layers. Together, they correspond to the "confined" water identified in the experiments.

The dynamics of the water molecules in the three layers are different both in respect to interlayer transitions and lateral motion within the layers. The former is quantified by determining average residence times for the water molecules in the layers. In a $1 \mathrm{~ns}$ time-window, they are found to be $\sim 31,54$, and $250 \mathrm{ps}$ for water molecules in the bulk-like, outer, and inner water layers, respectively. The lateral diffusion of the water molecules in each layer was determined by mean-square displacement calculations. They were used to infer diffusion constants of, $1.45 \times 10^{-5} \mathrm{~cm}^{2} \mathrm{~s}^{-1}, 4.95 \times 10^{-6}$ $\mathrm{cm}^{2} \mathrm{~s}^{-1}$, and $1.68 \times 10^{-6} \mathrm{~cm}^{2} \mathrm{~s}^{-1}$ for water in the bulk-like, outer, and inner water layers, respectively.

We note that the diffusion constant for the water molecules in the $\sim 10 \AA$ thick bulk-like water layer outside the membrane is about a factor of two smaller than for bulk water, which has an experimental and simulated diffusion constant of $\sim 2.33 \times 10^{-5} \mathrm{~cm}^{2} \mathrm{~s}^{-1}$. This result suggests that the electric field from the atoms in the head groups of the lipid molecules is strong enough to influence the dynamics significantly but is too weak to impose a change in the density. The diffusion constant for all water molecules, irrespective of their position in the membrane, was found to be $1.06 \times 10^{-5} \mathrm{~cm}^{2} \mathrm{~s}^{-1}$, which compares well with the mole-fraction weighted average of the diffusion constants for water in the different layers.

In Ref. 19, the dynamics of water in a POPC bilayer membrane at $310 \mathrm{~K}$ has been studied by MD simulations. The head group of the lipid is the same as in our study and the diffusion of water is therefore expected to be similar in the two systems. Diffusion constants have been determined for water molecules hydrogen-bonded to the carbonyl groups, phosphate groups, and amine groups (nitrogen) for at least 70 ps using a 100 ps time-window. Because the water molecules follow the motion of their respective groups in the lipid head group, their diffusion constants may be compared to ours in Table IV for the selected atoms. We see that there is a qualitative agreement of the relative magnitude of the diffusion constants for the selected atoms and groups, but the value of the diffusion constants in Ref. 19 is two orders of magnitude larger than ours. We attribute this discrepancy to the much shorter time-window ( $\sim 100 \mathrm{ps})$ used in Ref. 19 compared to our time-window of $2 \mathrm{~ns}$. Recall that diffusion constants for atoms in large molecules with rotational and intramolecular bounded motions depend on the time-window in mean-square displacement calculations, or equivalently, on the energy resolution and dynamic range in a scattering experiment. Only with time-windows large enough for all bounded motions to 
appear as bounded will a diffusion constant that is independent of the time-window be obtained.

The authors ${ }^{19}$ also studied the diffusion of other water molecules, less strongly bound to the lipid molecules, but a direct comparison with our results is difficult because they used different criteria in their selection of the water molecules. Qualitatively, however, both studies show that the diffusion constant of water decreases as one goes deeper into the membrane. The magnitude of the diffusion constants are also similar to ours. Because water is a small molecule and the time for the bounded rotational motion of the water molecule to manifest itself is very short, the translational diffusion constant inferred from their and our simulation should be comparable.

The decay rate in the number of water molecules originally in the first solvation shell around the nitrogen, phosphorus, and carbonyl carbon atoms suggests the existence of three types of water: loosely bound, bound with an intermediate strength, and strongly bound. If we take the binding strength of the water molecules to be proportional to their average residence time, then there is an increase in the bond strength of two orders of magnitude in going from the weakest to the strongest bound water molecules.

The incoherent scattering functions for selected atoms in the lipid head groups and for the water molecules were calculated at two wave vector transfers $q=0.5$ and $0.9 \AA^{-1}$ and compared to experiments. At both wave vectors, a twoLorentzian fit to the observed quasielastic spectra showed two time scales for the motion of the $\mathrm{H}$ atoms. An analysis of the observed intensities indicates that $\sim 7-10$ water molecules/lipid are bound so strongly to the lipids that they essentially follow their motion. A similar analysis of the simulation data showed that $\sim 6$ water molecules are strongly bound to the atoms in the lipids which compares with our upper bound of $\sim 8$ molecules obtained by our kinetic analysis. The simulations allow a more detailed investigation of the water dynamics as a function of their positions in the membrane than possible with scattering experiments which average over larger regions.

\section{ACKNOWLEDGMENTS}

This work was supported by the U.S. National Science Foundation under Grant Nos. DMR-0705974 and DGE1069091 and by the Danish Center for High Performance Computing, DTU, and SDU.

${ }^{1}$ J. Milhaud, Biochim. Biophys. Acta 1663, 19 (2004).

2J.-M. Zanotti, M.-C. Bellissent-Funel, and J. Parello, Biophys. J. 76, 2390 (1999)

${ }^{3}$ S. König, E. Sackmann, D. Richter, R. Zorn, C. Carlile, and T. M. Bayerl, J. Chem. Phys. 100, 3307 (1994).

${ }^{4}$ S. K. Pal, D. Sakul, D. Mandal, and K. Bhattacharyya, J. Phys. Chem. B 108, 4529 (2000).

${ }^{5}$ S. K. Pal and A. H. Zewail, Chem. Rev. 104, 2099 (2004).

${ }^{6}$ J. Swenson, F. Kargl, P. Berntsen, and C. Svanberg, J. Chem. Phys. 129, 045101 (2008).

${ }^{7}$ D. Russo, R. K. Murarka, G. Hura, E. Verschell, J. R. D. Copley, T. HeadGordon, J. Phys. Chem. B 108, 19885 (2004).

${ }^{8}$ M. R. Harpham, B. M. Ladanyi, N. E. Levinger, and K. W. Herwig, J. Chem. Phys. 121, 7855 (2004).

${ }^{9}$ W. Pfeiffer, T. Henkel, E. Sackmann, W. Knoll, and D. Richter, Europhys. Lett. 8, 201 (1989).
${ }^{10}$ S. König, W. Pfeiffer, T. Bayerl, D. Richter, and E. Sackmann, J. Phys. II 2, 1589 (1992).

${ }^{11}$ M. C. Rheinstädter, T. Seydel, F. Demmel, and T. Salditt, Phys. Rev. E 71, 061908 (2005).

${ }^{12}$ M. Hishada and K. Tanaka, Phys. Rev. Lett. 106, 158102 (2011).

${ }^{13}$ W. Zhao, D. E. Moilanen, E. E. Fenn, and M. D. Fayer, J. Am. Chem. Soc. 130, 13927 (2008).

${ }^{14}$ S. J. Marrink, M. Berkowitz, and H. J. C. Berendsen, Langmuir 9, 3122 (1993).

${ }^{15}$ K. Åman, E. Lindahl, O. Edholm, P. Håkansson, and P. O. Westlund, Biophys. J. 84, 102 (2003).

${ }^{16}$ H. I. Petrache, S. E. Feller, and J. F. Nagle, Biophys. J. 72, 2237 (1997).

${ }^{17}$ Z. Zhang and M. L. Berkowitz, J. Phys. Chem. B 113, 7676 (2009).

${ }^{18}$ V.A Makarov, M. Feig, B. K. Andrews, and B. M. Pettitt, Biophys. J. 75, 150 (1998).

${ }^{19}$ T. Róg, K. Murzyn, and M. Pasenkiewicz-Gierula, Chem. Phys. Lett. 352, 323 (2002).

${ }^{20}$ M. Bai, A. Miskowiec, F. Y. Hansen, H. Taub, T. Jenkins, M. Tyagi, S. O. Diallo, E. Mamontov, K. W. Herwig, and S.-K. Wang, Europhys. Lett. 98, 48006 (2012).

${ }^{21}$ M. Bai, A. Miskowiec, P. Schnase, G. King, F. Y. Hansen, H. Taub, T. Heitmann, P. Miceli, H. Kaiser, M. Dubey, S. Singh, and J. Majewski, "Study of single-supported DMPC lipid bilayer membranes as a function of hydration level using neutron reflectivity and atomic force microscopy," (unpublished).

${ }^{22}$ J. B. Klauda, N. Kucerca, B. R. Brooks, R. W. Pastor, and J. F. Nagle, Biophysics. J. 90, 2796 (2006).

${ }^{23}$ J. Sonne, M. Ø. Jensen, F. Y. Hansen, L. Hemmingsen, and G. H. Peters, Biophys. J. 92, 4157 (2007).

${ }^{24}$ J. B. Klauda, R. M. Venable, J. A. Freites, J. W. O’Connor, D. J. Tobias, C. Mondragon-Ramirez, I. Vorobyov, A. D. MacKerell, Jr., and R. W. Pastor, J. Phys. Chem. B 114, 7830 (2010).

${ }^{25}$ W. L. Jorgensen, J. Chandrasekhar, J. D. Medura, R. W. Impey, and M. L. Klein, J. Chem. Phys. 79, 926 (1983).

${ }^{26}$ L. Kale, R. Skeel, M. Bhandarkar, R. Brunner, A. Gursoy, N. Krawetz, J. Phillips, A. Shinozaki, K. Varadarajan, and K. Schulten, J. Comput. Phys. 151, 283 (1999).

${ }^{27}$ S. E. Feller, Y. Zhang, R. W. Pastor, and B. R. Brooks, J. Chem. Phys. 103, 4613 (1995).

${ }^{28}$ U. Essmann, L. Perera, M. L. Berkowitz, T. Darden, H. Lee, and L. G. Pedersen, J. Chem. Phys. 103, 8577 (1995).

${ }^{29}$ J. F. Nagle, R. Zhang, S. Tristram-Nagle, H. I. Petrache, and R. M. Suter, Biophys. J. 70, 1419 (1996).

${ }^{30}$ N. Kucerka, Y. Liu, N. Chu, H. I. Petrache, S. Tristram-Nagle, and J. F. Nagle, Biophys. J. 88, 2626 (2005).

${ }^{31}$ M. Tanaka, G. Girard, R. Davis, A. Peuto, and N. Bignell, Metrologia 38, 301 (2001).

${ }^{32}$ K. O. Evans, Int. J. Mol. Sci. 9, 498 (2008).

${ }^{33}$ J. F. Nagle and S. Tristram-Nagle, Biochim. Biophys. Acta 1469, 159 (2000).

${ }^{34}$ W. Marshall and S. W. Lovesey, Theory of Thermal Neutron Scattering (Oxford University Press, Oxford, 1971).

${ }^{35}$ M. W. Mills, J. Phys. Chem. 77, 685 (1973); K. Krynicki, C. D. Green, and D. W. Sawyer, Fraday Discuss. Chem. Soc. 66, 199 (1978); K. T. Gillen, D. C. Douglass, and M. J. R. Hoch, J. Chem. Phys. 57, 5117 (1973); C. A. Angell, E. D. Finch, L. A. Wolf, and P. Bach, ibid. 65, 3063 (1976); L. A. Wolf, J. Chem. Soc., Faraday Trans. 1 71, 784 (1975).

${ }^{36}$ F. Y. Hansen and H. Taub, Commun. Comput. Phys. 6, 231 (2009).

${ }^{37}$ M. Abramowitz and I. Stegun, Handbook of Mathematical Functions (Dover, New York, 1970).

${ }^{38}$ A. D. Enevoldsen, F. Y. Hansen, A. Diama, H. Taub, R. M. Dimeo, D. A. Neumann, and J. R. D. Copley, J. Chem. Phys. 126, 104704 (2007).

${ }^{39}$ M. Bee, Quasielastic Neutron Scattering (Hilger, Bristol/Philadelphia, 1988).

${ }^{40}$ A. Mayer, R. Dimeo, P. Gehring, and D. Neumann, Rev. Sci. Instrum. 74, 2759 (2003).

${ }^{41}$ E. Mamontov and K. W. Herwig, Rev. Sci. Instrum. 82, 085109 (2011).

${ }^{42}$ S.-K. Wang, E. Mamontov, M. Bai, F. Y. Hansen, H. Taub, J. R. D. Copley, V. García, G. Gasparovic, T. Jenkins, M. Tyagi, K. W. Herwig, D. Neumann, W. Montfrooij, and U. G. Volkmann, Europhys. Lett. 91, 66007 (2010).

${ }^{43}$ F. Y. Hansen, L. Criswell, D. Fuhrmann, K. W. Herwig, A Diama, R. M. Dimeo, D. A. Neumann, U. G. Volkmann, and H. Taub, Phys. Rev. Lett. 92, 046103 (2004). 